\title{
Secular Dynamics around small bodies with Solar Radiation Pressure
}

\author{
J. Feng, X.-Y. Hou* \\ School of Astronomy and Spacec Sciences, Nanjing University, China
}

\begin{abstract}
This study focuses on the secular dynamics of orbital motion around asteroids with joint effects of $C_{20}$ term and solar radiation pressure (SRP). With their combined influences, two specific orbits are firstly studied, namely the solar terminator orbit (STO) and the heliotropic orbit. Then, the global orbital motion other than the two specific ones is investigated. Finally, the influence of the asteroid's orbital eccentricity around the Sun on the secular dynamics is discussed.

Keywords: numerical, celestial mechanics, minor planets, asteroids
\end{abstract}

\section{Introduction}

With combined effects of the gravity and the SRP, the secular dynamics of orbital motion in the vicinity of celestial bodies has been a research focus for many years, as a result of but not limited to the following reasons: (1) particles/dusts close to or on the surface of the bodies are significantly affected by the SRP. They might escape from, collide with or stay for long time near the body [3, 10, 11, 19 , 22, 26]; (2) small bodies in the solar system have become popular targets for deep

\footnotetext{
*Corresponding author

Email address: silence@nju.edu.cn (X.-Y. Hou )
} 
space explorations world widely. Weak gravity of these bodies and large areato-mass ratio of the spacecraft requires the consideration of the joint effects for mission orbit design and control, etc. [5, 7, 27, 29, 34]; (3) the space debris have become an emergent research topic because of its exponential rate of increase and potential hazards to spacecraft. For the high area-to-mass ratio and high-altitude debris, their orbital motion is strongly affected by the SRP [4, 6, 31, 32, 35].

Using the point-mass gravity of the body, only considering the first-order effect of the SRP, and ignoring the body's orbital eccentricity around the Sun, Mignard [20] found that the averaged dynamics around the body is integrable and gave the integral in Keplerian elements [21]. Considering the body's orbital eccentricity and using the true anomaly instead of the time as the independent variables, Scheeres [27] proved that the equations of motion (EOM) can be simplified to the same form as those for case of the circular orbit. Using the averaged system, Richter and Keller [23] gave a simplified description of the solution based on a constant vector and two vectors spinning around it. Using the eccentricity vector and the angular momentum vector, Scheeres [29], Rosengren and Scheeres [24] derived the time explicit form of the solution, which is useful for practical applications.

With the joint effects of the SRP and the body's point-mass gravity, there are two specific kinds of orbits. The first type is the STO, whose inclination is around $90^{\circ}$ and the orbital plane points toward or away from the Sun [30, 13]. STO is stable and allows stable quasi-periodic motion in its vicinity [2]. Actually, the STO is the halo family bifurcating from the planar Lyapunov family around the L2 point of the circular restricted three body problem (CRTBP) or Hill problem 
[1, 2, 9, 18, 14]. The other type is the Heliotropic orbit, whose orbital plane completely lies in the small body's orbital plane around the Sun and whose perigee always points toward or away from the Sun. It is not always stable and has been extensively studied for the problem of particle's motion near planets [20, 10,11 , 26, 12]. Recently, its potential applications in asteroid explorations [16, 25] and in Earth missions [6] have attracted researchers' attentions.

This study focuses on the orbital motion around the small solar system bodies. Because of their highly irregular shapes, the effect of non-spherical gravity field (more specifically, the zonal terms) on the long-term behaviors of the orbital motion around them cannot be ignored for motions not far away from the asteroids. Although there are studies on the long-term joint effects between nonspherical terms and the third body perturbations [35, 4], there are much less work on the long-term dynamics between non-spherical terms and the SRP. Scheeres [28] briefly discussed the influence of the second order and degree gravitational field on the stability of the STO. Lantukh et al. [16] shows that most non-planar frozen families (including the STO) are unstable when the $C_{20}$ term and the SRP are simultaneously considered. In this paper, our investigation on the STO shows that even very small value of the $C_{20}$ term introduces instability to the orbital motion. The divergence speed of the STO increases with the $C_{20}$ values, therefore, the STO seems to be 'stable' within short-time scales if $C_{20}$ is small. Interestingly, if $C_{20}$ increases larger than a critical value, the orbit becomes stable instead (see Section 3.2 for details). This is a phenomenon never pointed out before. For prograde and retrograde Heliotropic orbit, some interesting phenomena are discovered by applying a different methodology from previous studies [16, 25] (see 
Section 4 for details). And a better criterion for their stability is proposed. In addition to these two specific kinds of orbits, global dynamics is discussed for two subcases, i.e. the SRP dominant case and the $C_{20}$ dominant case, respectively. Finally, the effect of the small body's orbit eccentricity around the Sun is briefly discussed.

The area-to-mass ratio used in this study ranges from 0.01 to $0.03 \mathrm{~kg} \cdot \mathrm{m}^{-2}$, corresponding to typical values of the spacecraft around a small body [34]. The radius of the small body is taken between $500 \mathrm{~m}$ and $1 \mathrm{~km}$. Finally, to simplify the model, the shadow effect of the SRP is not considered in this study.

\section{Dynamical Modelling}

\subsection{Solar Radiation Pressure}

Denoting the area-to-mass ratio of the spacecraft as $S / m$, the solar radiation flux at a reference distance $r_{a}$ from the Sun as $\rho_{\odot}$, the light speed as $c$, and the albedo of the asteroid as $\kappa$, the EOM of the spacecraft in the asteroid-centered inertial frame, shown in the left plot of Fig.1, is given as

$$
\boldsymbol{R}^{\prime \prime}=\frac{d^{2} \boldsymbol{R}}{d t^{2}}=-G m_{a} \frac{\boldsymbol{r}}{r^{3}}-(1+\kappa) \frac{\rho_{\odot} r_{a}^{2}}{c} \frac{S}{m} \frac{\boldsymbol{\Delta}}{\Delta^{3}}
$$

in which $m_{a}$ is the mass of the asteroid and $G$ is the gravitational constant. Taking the unit of length, mass and time as

$$
[L]=a_{e},[M]=m_{a},[T]=\sqrt{\frac{[L]^{3}}{G[M]}}
$$



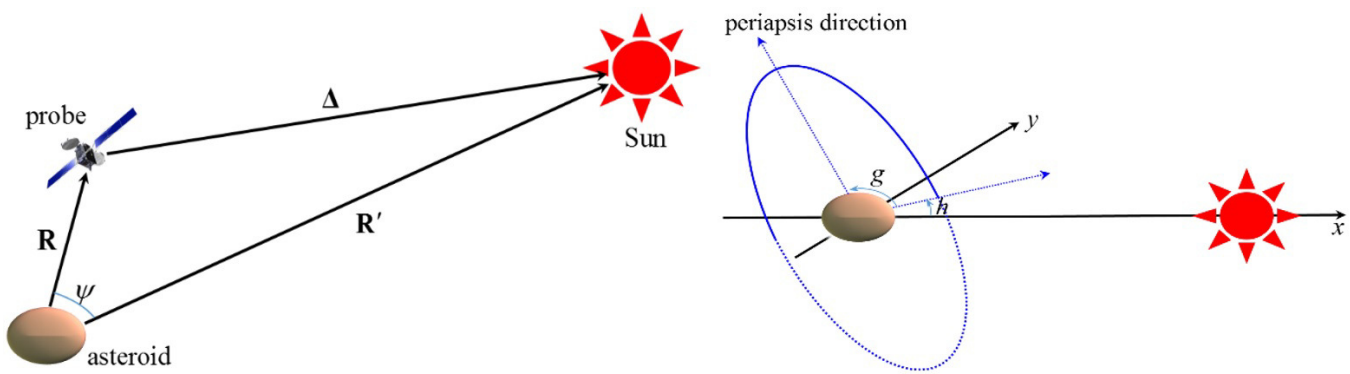

Figure 1: Left: The illustration of SRP; Right: Sketch of the asteroid-centered frame rotating with the Sun and the angles $g$ and $h$ in $\mathrm{Eq} 18$

where $a_{e}$ a symbol indicating the reference radius of the asteroid used as the length unit. In our work, it is defined by following Eq 21 . Introducing the following non-dimensional quantities

$$
\begin{gathered}
\boldsymbol{r}=\frac{\boldsymbol{R}}{[L]}, \boldsymbol{r}^{\prime}=\frac{\boldsymbol{R}^{\prime}}{[L]}, \boldsymbol{\delta}=\frac{\boldsymbol{\Delta}}{[L]}, \tau=\frac{t}{[T]} \\
\ddot{\boldsymbol{r}}=\frac{d^{2} \boldsymbol{r}}{d \tau^{2}}=\frac{[T]^{2} d^{2} \boldsymbol{r}}{d t^{2}}=\frac{[T]^{2}}{[L]} \frac{d^{2} \boldsymbol{R}}{d t^{2}}=\frac{[T]^{2}}{[L]} \boldsymbol{R}^{\prime \prime}
\end{gathered}
$$

and substituting them into $\mathrm{Eq} 1$, a non-dimensional form of Eq.1 can be written as

$$
\ddot{\boldsymbol{r}}=-\frac{\boldsymbol{r}}{r^{3}}-\beta \odot \frac{\boldsymbol{\delta}}{\delta^{3}}=\frac{\partial V_{0}}{\partial \boldsymbol{r}}+\frac{\partial V_{1}}{\partial \boldsymbol{r}}
$$

in which

$$
V_{0}=\frac{1}{r}, V_{1}=-\frac{\beta_{\odot}}{\delta}, \beta_{\odot}=(1+\kappa) \frac{\rho_{\odot} r_{a}^{2}}{c} \frac{S}{m} \frac{a_{e}^{2}}{G m_{a}}
$$


and the SRP can be expanded in the following form

$$
V_{1}=-\frac{\beta_{\odot}}{\Delta}=-\frac{\beta_{\odot}}{r^{\prime}} \sum_{n=0}^{\infty}\left(\frac{r}{r^{\prime}}\right)^{n} P_{n}(\cos \psi)
$$

The term with $n=0$ is a constant, and the terms with $n \geq 2$ are very small. Therefore, they are both ignored in this study and only the term with $n=1$ is taken into account. The simplified SRP is written as

$$
V_{1} \approx-\beta_{\odot} \frac{r}{r^{\prime 2}} \cos \psi=-\beta_{\odot} \frac{\boldsymbol{r} \cdot \boldsymbol{r}^{\prime}}{r^{\prime 3}}
$$

\subsection{The $C_{20}$ and $C_{22}$ terms}

In the inertial frame with origin at the asteroid's barycenter and reference plane as the equatorial plane, the potential function of the $C_{20}$ and $C_{22}$ terms (where $S_{22}=0$ by selecting the proper coordinate) are usually expressed in Keplerian orbital elements of the motion around the asteroid, i.e. the inclination $i$, the longitude of the acsending node $\Omega$, the argument of the pericenter $\omega$ and the true anomaly $f$.

$$
\begin{aligned}
V_{20}= & -\frac{C_{20}}{2 r^{3}}\left[\left(1-\frac{3}{2} \sin ^{2} i\right)+\frac{3}{2} \sin ^{2} i \cos 2 u\right] \\
V_{22}= & \frac{3 C_{22}}{4 r^{3}}\left[(1+\cos i)^{2} \cos \left(2 u+2 \Omega_{e}\right)\right. \\
& \left.+(1-\cos i)^{2} \cos \left(2 u-2 \Omega_{e}\right)+2 \sin ^{2} i \cos 2 \Omega_{e}\right]
\end{aligned}
$$

in which $u=f+\omega$ and $\Omega_{e}$ is defined as

$$
\Omega_{e}=\left(\Omega-S_{0}\right)-n_{e}\left(t-t_{0}\right)
$$


where $n_{e}$ is the rotation rate of the asteroid, and $S_{0}$ is the initial phase angle of the $x$-axis of the asteroid's body-fixed frame with respect to the inertial frame at initial time epoch $t_{0}$.

\subsection{The averaged dynamics}

From the orbital geometry, it is known that

$$
\begin{aligned}
& \cos \psi=A \cos f+B \sin f \\
A & =\frac{1}{4}(1-\cos i)\left[\left(1+\cos i^{\prime}\right) \cos \left(\omega-\theta+u^{\prime}\right)\right. \\
& \left.+\left(1-\cos i^{\prime}\right) \cos \left(\omega-\theta-u^{\prime}\right)\right] \\
& +(1+\cos i)\left[\left(1+\cos i^{\prime}\right) \cos \left(\omega+\theta-u^{\prime}\right)\right. \\
& \left.+\left(1-\cos i^{\prime}\right) \cos \left(\omega+\theta+u^{\prime}\right)\right] \\
& +2 \sin i \sin i^{\prime}\left[\cos \left(\omega-u^{\prime}\right)-\cos \left(\omega+u^{\prime}\right)\right] \\
B & =-\frac{1}{4}(1-\cos i)\left[\left(1+\cos i^{\prime}\right) \sin \left(\omega-\theta+u^{\prime}\right)\right. \\
& \left.+\left(1-\cos i^{\prime}\right) \sin \left(\omega-\theta-u^{\prime}\right)\right] \\
& +(1+\cos i)\left[\left(1+\cos i^{\prime}\right) \sin \left(\omega+\theta-u^{\prime}\right)\right. \\
& \left.+\left(1-\cos i^{\prime}\right) \sin \left(\omega+\theta+u^{\prime}\right)\right] \\
& +2 \sin i \sin i^{\prime}\left[\sin \left(\omega-u^{\prime}\right)-\sin \left(\omega+u^{\prime}\right)\right]
\end{aligned}
$$

in which

$$
\theta=\Omega-\Omega^{\prime}, u^{\prime}=f^{\prime}+\omega^{\prime}
$$

and $i^{\prime}, f^{\prime}, \Omega, \omega^{\prime}$ are respectively the orbit inclination, true anomaly, longitude of the ascending node, argument of perigee of the Sun's orbit w.r.t. the asteroid. 
Taking the asteroid's heliocentric orbital plane as the reference plane, we have $i^{\prime}, \Omega^{\prime}=0$. Further, ignoring the asteroid's orbital eccentricity $e^{\prime}$ and argument of perigee $\omega^{\prime}$, Eq.10 can be simplified as follows

$$
\begin{aligned}
A & =\frac{1}{4}\left[2(1-\cos i) \cos \left(\omega-\Omega+M^{\prime}\right)\right. \\
& \left.+2(1+\cos i) \cos \left(\omega+\Omega-M^{\prime}\right)\right] \\
B & =-\frac{1}{4}\left[2(1-\cos i) \sin \left(\omega-\Omega+M^{\prime}\right)\right. \\
& \left.+2(1+\cos i) \sin \left(\omega+\Omega-M^{\prime}\right)\right]
\end{aligned}
$$

Substituting Eq 9 into Eq.6 and then averaging, it can be obtained that

$$
\left\langle V_{1}\right\rangle=\frac{1}{T} \int_{0}^{T} V_{1} d t=\frac{3}{2} \tilde{\beta}_{\odot} e a A
$$

in which $T=a^{-\frac{3}{2}}$ is the non-dimensional period of the Keplerian orbit. $a$ and $e$ are the semi-major axis and orbit eccentricity of the Keplerian orbit, and

$$
\tilde{\beta}_{\odot}=\frac{\beta_{\odot}}{a^{\prime 2}}=(1+\kappa) \frac{\rho_{\odot}}{c}\left(\frac{r_{a}}{a^{\prime}}\right)^{2} \frac{S}{m} \frac{a_{e}^{2}}{G m_{a}}
$$

For a fast-rotating asteroid, the $\Omega_{e}$ in Eq 8 can be treated as a fast variable. Therefore $\left\langle V_{22}\right\rangle=0$, and the average of $V_{20}$ is given as

$$
\left\langle V_{20}\right\rangle=\frac{C_{20}}{4 a^{3}}\left(1-e^{2}\right)^{-\frac{3}{2}}\left(1-3 \cos ^{2} i\right)
$$

Using the Delaunay variables

$$
\begin{gathered}
\tilde{l}=M, \tilde{g}=\omega, \tilde{h}=\Omega \\
\tilde{L}=\sqrt{a}, \tilde{G}=\tilde{L} \sqrt{1-e^{2}}, \tilde{H}=\tilde{G} \cos i
\end{gathered}
$$


the Hamiltonian of the system is expressed as

$$
\begin{aligned}
H & =-\frac{1}{2 \tilde{L}^{2}}-\left\langle V_{1}\right\rangle-\left\langle V_{20}\right\rangle \\
& =-\frac{1}{2 \tilde{L}^{2}}-\frac{C_{20}}{4 \tilde{L}^{3} \tilde{G}^{3}}\left(1-\frac{3 \tilde{H}^{2}}{\tilde{G}^{2}}\right)-\frac{3}{4} \tilde{\beta_{\odot}} \tilde{L} \sqrt{\tilde{L}^{2}-\tilde{G}^{2}} \\
& {\left[\left(1-\frac{\tilde{H}}{\tilde{G}}\right) \cos \left(\tilde{g}-\tilde{h}+M^{\prime}\right)+\left(1+\frac{\tilde{H}}{\tilde{G}}\right) \cos \left(\tilde{g}+\tilde{h}-M^{\prime}\right)\right] }
\end{aligned}
$$

With the following canonical transform

$$
\begin{gathered}
l=\tilde{l}, g=\tilde{g}, h=\tilde{h}-M^{\prime}=h-n^{\prime} t \\
L=\tilde{L}, G=\tilde{G}, H=\tilde{H}
\end{gathered}
$$

the $x$-axis of the asteroid's body-fixed frame is transformed to the direction pointing towards the Sun, as indicated in the right frame of Fig.1. The Hamilton function is then transformed to

$$
\begin{aligned}
& K=-\frac{1}{2 L^{2}}-\frac{C_{20}}{4 L^{3} G^{3}}\left(1-\frac{3 H^{2}}{G^{2}}\right)-\frac{3}{4} \tilde{\beta}_{\odot} L \sqrt{L^{2}-G^{2}} \\
& \quad\left[\left(1-\frac{H}{G}\right) \cos (g-h)+\left(1+\frac{H}{G}\right) \cos (g+h)\right]-n^{\prime} H
\end{aligned}
$$

As the reference plane is defined as the asteroid's equatorial plane and Eq 13 is derived by assuming the $x-y$ plane as the asteroid's orbital plane, Eq 19 is only valid when the equatorial plane and orbital plane of the asteroid coincide with each other, namely the asteroid's obliquity is $0^{\circ}$ or $180^{\circ}$. According to Lantukh et al. [16], about 25 percent of small asteroids (diameter $<30 \mathrm{~km}$ ) possess this property, which is related to the YORP effect Vokrouhlicky et al. [33]. The EOM 
corresponding to the dynamical system expressed by Eq.19 is given as

$$
\begin{aligned}
\dot{g} & =\frac{3 C_{20}}{4 L^{3} G^{4}}\left(1-\frac{5 H^{2}}{G^{2}}\right) \\
& +\frac{3}{4} \tilde{\beta}_{\odot} \frac{L G}{\sqrt{L^{2}-G^{2}}}\left[\left(1-\frac{H}{G}\right) \cos (g-h)+\left(1+\frac{H}{G}\right) \cos (g+h)\right] \\
& -\frac{3}{4} \tilde{\beta}_{\odot} \frac{L H \sqrt{L^{2}-G^{2}}}{G^{2}}[\cos (g-h)-\cos (g+h)] \\
\dot{h} & =\frac{3 C_{20}}{2 L^{3} G^{4}} \frac{H}{G}+\frac{3}{4} \tilde{\beta}_{\odot} \frac{L \sqrt{L^{2}-G^{2}}}{G}[\cos (g-h)-\cos (g+h)]-n^{\prime} \\
\dot{G} & =-\frac{3}{4} \tilde{\beta}_{\odot} L \sqrt{L^{2}-G^{2}}\left[\left(1-\frac{H}{G}\right) \sin (g-h)+\left(1+\frac{H}{G}\right) \sin (g+h)\right] \\
\dot{H} & =\frac{3}{4} \tilde{\beta}_{\odot} L \sqrt{L^{2}-G^{2}}\left[\left(1-\frac{H}{G}\right) \sin (g-h)-\left(1+\frac{H}{G}\right) \sin (g+h)\right]
\end{aligned}
$$

\subsection{Statement}

Before proceeding to further details, we make a short statement here. The triaxial shape model with semi axes $a \geq b \geq c$ for the asteroid is used. The density is $\rho$ in this study, which takes the value of $2000 \mathrm{~kg} \cdot \mathrm{m}^{-3}$. The reference radius in this study is taken as

$$
a_{e}=(a b c)^{1 / 3}=a(\beta \gamma)^{1 / 3}, \beta=b / a, \gamma=c / a
$$

where $\beta$ and $\gamma$ are shape parameters normalized by $a$. The $C_{20}$ and $C_{22}$ terms can be obtained as

$$
\begin{aligned}
& C_{20}=\frac{2 c^{2}-a^{2}-b^{2}}{10 a_{e}^{2}}=\frac{a^{2}}{a_{e}^{2}} \frac{\left(2 \gamma^{2}-\beta^{2}-1\right)}{10} \\
& C_{22}=\frac{a^{2}-b^{2}}{20 a_{e}^{2}}=\frac{a^{2}}{a_{e}^{2}} \frac{1-\beta^{2}}{20}
\end{aligned}
$$


from which, it is easily to get that

$$
a=\frac{a_{e}}{(\beta \gamma)^{1 / 3}}, C_{20}=\frac{\left(2 \gamma^{2}-\beta^{2}-1\right)}{10(\beta \gamma)^{2 / 3}}, C_{22}=\frac{1-\beta^{2}}{20(\beta \gamma)^{2 / 3}}
$$

According to $\mathrm{Eq}, 14$, for fixed values of $S / m$ and the relative perturbative strength of the SRP with respect to the asteroid gravity is determined by the value of $a_{e}$. Fixing the value of $a_{e}$, the values of $C_{20}$ and $C_{22}$ terms can be varied by simutaneously adjusting the shape parameters $\beta, \gamma$.

\section{STO}

According to the research of Mignard and Henon [21], Richter and Keller [23], and Scheeres [27], the system of Eq.19 is completely integrable if only considering the SRP (i.e. $C_{20}=0$ ). Scheeres et al. [30] found two types of equilibrium points (EPs), which correspond repectively to the STO and the Heliotropic orbit that are described in the Introduction. Before preceding to further details, we make clear that we are dealing with the averaged Hamiltonian Eq,19, i.e. the mean (or the averaged) but not the instantaneous orbital elements of the orbits. As a result, an $\mathrm{EP}$ of $\mathrm{Eq} 20$ only means that the averaged orbit remains fixed w.r.t. the Sun-asteroid line (see Eq 18 . In the physical space, the orbit is still moving around the asteroid because the mean anomaly $M$ (which does appear in Eq20 due to the averaging process) constantly changes with time. This section will discuss the effect of the $C_{20}$ term on the STO, and leave the Heliotropic orbit to the next section. 
Table 1: Different combinations of $g$ and $h$ satisfying Eq 25

\begin{tabular}{c|cccccccc}
\hline Situation & 1 & 2 & 3 & 4 & 5 & 6 & 7 (STO) & 8 (STO) \\
\hline$g$ & 0 & $\pi$ & $\pi$ & 0 & $\pi / 2$ & $-\pi / 2$ & $\pi / 2$ & $-\pi / 2$ \\
$h$ & 0 & $\pi$ & 0 & $\pi$ & $-\pi / 2$ & $\pi / 2$ & $\pi / 2$ & $-\pi / 2$ \\
\hline
\end{tabular}

As an EP of Eq,20, the STO satisfies

$$
\dot{g}=\dot{h}=\dot{G}=\dot{H}=0
$$

According to Eq 20, the last two equalities of Eq $24(\dot{G}=\dot{H}=0)$ require

$$
\left(1-\frac{H}{G}\right) \sin (g-h)=0,\left(1+\frac{H}{G}\right) \sin (g+h)=0
$$

Since the STO is non-planar, it is obvious that $1 \mp H / G \neq 0$, which means $\sin (g \mp h)=0$. Assuming $g \in(-\pi, \pi], h \in(-\pi, \pi]$, several possible cases of $g, h$ values are given in Table 1 . However, for some specific combinations of $C_{20}$ and $\tilde{\beta}_{\odot}$, not all the situations in Table 1 can make the first two equalities of Eq. 24 For cases 1,2 (or 3,4$)$ in Table 1 , the following equations can be obtained

$$
\begin{aligned}
& \dot{g}=f_{1}(G, H)=\frac{3 C_{20}}{4 L^{3} G^{4}}\left(1-\frac{5 H^{2}}{G^{2}}\right) \pm \frac{3}{2} \tilde{\beta}_{\odot} \frac{L G}{\sqrt{L^{2}-G^{2}}}=0 \\
& \dot{h}=f_{2}(G, H)=\frac{3 C_{20}}{2 L^{3} G^{4}} \frac{H}{G}-n^{\prime}=0
\end{aligned}
$$

in which the upper and lower signs correspond to cases 1, 2 and 3, 4 respectively. For cases 5, 6 (or 7, 8) in Table1, the following equations can be obtained

$$
\begin{aligned}
& \dot{g}=f_{1}(G, H)=\frac{C_{20}}{2 L^{6} G^{2}}\left(1-\frac{5 H^{2}}{G^{2}}\right) \pm \tilde{\beta}_{\odot} \frac{H}{\sqrt{L^{2}-G^{2}}}=0 \\
& \dot{h}=f_{2}(G, H)=\frac{3 C_{20}}{2 L^{3} G^{4}} \frac{H}{G} \mp \frac{3}{2} \tilde{\beta}_{\odot} \frac{L \sqrt{L^{2}-G^{2}}}{G}-n^{\prime}=0
\end{aligned}
$$


in which the upper and lower sign correspond to situations 5-6 and 7-8 respectively. Since $C_{20}<0$, cases 3, 4 are always impossible, and cases 1, 2, 5, 6 are possible depending on the values of $C_{20}$ and $\tilde{\beta}_{\odot}$. The stability of the EP for

cases $1,2,5,6$ also changes with the values of $C_{20}$ and $\tilde{\beta}_{\odot}$. Readers can refer to the work of Lantukh et al. [16] for more details on these EPs. Cases 7, 8 are always possible, and they are the STOs which are extensively studied by previous researchers and also the focus of this section.

\subsection{Eccentricity and inclination}

When $C_{20}=0$, it can be obtained from $\mathrm{Eq} 27$ that $H=0$, namely the orbital inclination is $90^{\circ}$. From the second equation of Eq.27, it can be derived

$$
G=\left(L^{2}+\left(\frac{2 n^{\prime}}{3 \tilde{\beta}_{\odot}}\right)^{2}\right)^{-1 / 2} L^{2}
$$

Similar with the definition in Mignard and Henon [21], Scheeres [29], the solar radiation angle $\Lambda$ is introduced as

$$
\tan \Lambda=\frac{3 \tilde{\beta}_{\odot} L}{2 n^{\prime}}
$$

Then Eq.29 is actually equal to

$$
e=\cos \Lambda
$$

which indicates that the orbital eccentricity and inclination are constant for the given orbital radius $r_{a}$ of the asteroid and $S / m$ of the spacecraft. However, the situation is different after taking into account the $C_{20}$ term. Taking $a_{e}=500 \mathrm{~m}$ and $r_{a}=1 A U$ as an example, Fig 2 gives the variations of $e=\sqrt{1-(G / L)^{2}}$ as 

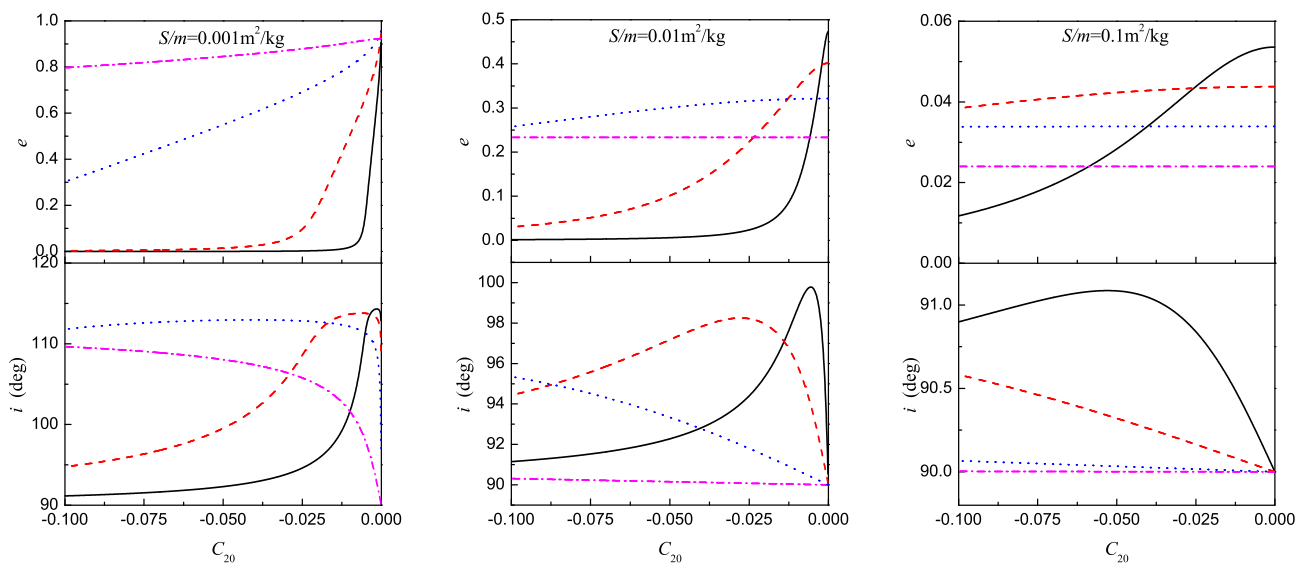

Figure 2: Curves of $e$ and $i$ of STO as a function of the $C_{20}$ term for $S / m=0.001,0.01,0.1$ $\mathrm{kg}^{-1} \cdot \mathrm{m}^{2}$, respectively. In each plot, the black, red dashed, blue dot and pinkish red dashed dot lines represent $a=2,3,5,10$, respectively.

a function of the $C_{20}$ term for $S / m=0.001,0.01,0.1 \mathrm{~kg}^{-1} \cdot \mathrm{m}^{2}$, respectively. It can be observed that:

1. With the increase of the $C_{20}$ term, the eccentricity of the STO decreases in general. However, the decreasing tendency of $e$ is less obvious when the orbital altitude is high, as the influence of the $C_{20}$ term weakens with the increasing orbital altitude;

2. The introduction of the $C_{20}$ term makes the inclination of STO larger than $90^{\circ}$. For high-altitude orbit, the inclination increases with the increase of the $C_{20}$ term, but this phenomenon is not obvious with the increase of orbital altitude. For low-altitude orbits, the inclination does not change monotonously as a function of the $C_{20}$ term. 


\subsection{Orbital Stability}

Denoting $\boldsymbol{X}=(g, h, G, H), \mathrm{Eq} 20$ can be written in the following form

$$
\dot{\boldsymbol{X}}=F(\boldsymbol{X})
$$

which can be expanded at the EP denoted as $X_{e}$ (i.e. the STO with $g=h=$ $\pi / 2$ and $G, H$ given by $\mathrm{Eq} 27$. Only considering the linearized term, the linearized equation can be obtained as

$$
\Delta \dot{\boldsymbol{X}}=A \Delta \boldsymbol{X}=\left.\frac{\partial F(\boldsymbol{X})}{\partial \boldsymbol{X}}\right|_{\boldsymbol{X}=\boldsymbol{X}_{e}} \Delta \boldsymbol{X}
$$

in which matrix $\boldsymbol{A}$ is a $4 \times 4$ matrix

$$
\boldsymbol{A}=\left[\begin{array}{cccc}
a_{11} & a_{12} & a_{13} & a_{14} \\
a_{21} & a_{22} & a_{23} & a_{24} \\
a_{31} & a_{32} & a_{33} & a_{34} \\
a_{41} & a_{42} & a_{43} & a_{44}
\end{array}\right]
$$

The expressions of $a_{i j}, i, j=1 \ldots 4$ are given in Appendix. For $g=h=\pi / 2$, we have $a_{11}=a_{12}=a_{21}=a_{22}=0$ and $a_{33}=a_{34}=a_{43}=a_{44}=0$. The eigenvalues of $A$ satisfy

$$
s(\lambda)=\lambda^{4}+\bar{b} \lambda^{2}+\bar{c}=0
$$

in which

$$
\begin{aligned}
& \bar{b}=-\left(a_{23} a_{32}+a_{24} a_{42}+a_{13} a_{31}+a_{14} a_{41}\right) \\
& \bar{c}=a_{13} a_{24}\left(a_{31} a_{42}-a_{41} a_{32}\right)-a_{14} a_{23}\left(a_{31} a_{42}-a_{41} a_{32}\right)
\end{aligned}
$$


It can be seen that the sign of $\bar{c}-\bar{b}^{2} / 4$ determines the eigenvalues of the linearized system. For $C_{20}=0$, it is easily obtained that $\bar{c}-\bar{b}^{2} / 4=0$. The system has two pairs of roots with the same eigenvalues

$$
\lambda_{1,2}=\lambda_{3,4}= \pm i \omega= \pm i\left(\frac{n^{\prime}}{\cos \Lambda}\right)
$$

In general, the solution of Eq 34 in this case should include Poisson terms in the form of $t \cos \lambda_{i} t$ or $t \sin \lambda_{i} t$, but the situation here is particular. Eq.20 in this situation is explicitly written as

$$
\begin{aligned}
\Delta \dot{g} & =-\frac{3}{2} \tilde{\beta}_{\odot} \frac{L^{3}}{G^{2}} \sqrt{L^{2}-G^{2}} \cdot \Delta H, \Delta \dot{h}=-\frac{3}{2} \tilde{\beta}_{\odot} \frac{L^{3}}{G^{2}} \sqrt{L^{2}-G^{2}} \cdot \Delta G \\
\Delta \dot{G} & =\frac{3}{2} \tilde{\beta}_{\odot} L \sqrt{L^{2}-G^{2}} \cdot \Delta h, \Delta \dot{H}=\frac{3}{2} \tilde{\beta}_{\odot} L \sqrt{L^{2}-G^{2}} \cdot \Delta g
\end{aligned}
$$

It is observed that for this specific situation this two-dimensional linearized system is completely separated and its solution can be written in the following form

$$
\begin{aligned}
& \Delta g=\alpha \cos \left(\omega t+\phi_{1}\right), \Delta H=\kappa \alpha \sin \left(\omega t+\phi_{1}\right) \\
& \Delta h=\beta \cos \left(\omega t+\phi_{2}\right), \Delta G=\kappa \beta \sin \left(\omega t+\phi_{2}\right)
\end{aligned}
$$

in which

$$
\kappa=L \sin \Lambda \cos \Lambda
$$

The frequency $\omega$ is given by Eq, 36 . Eq. 38 indicates that the STO is stable, which is coincident with the conclusions of previous research [21, 29]. However, for $C_{20} \neq 0$, the stability of the STO changes. Fixing $a_{e}=500 \mathrm{~m}$ and $\mathrm{S} / \mathrm{m}=$ 

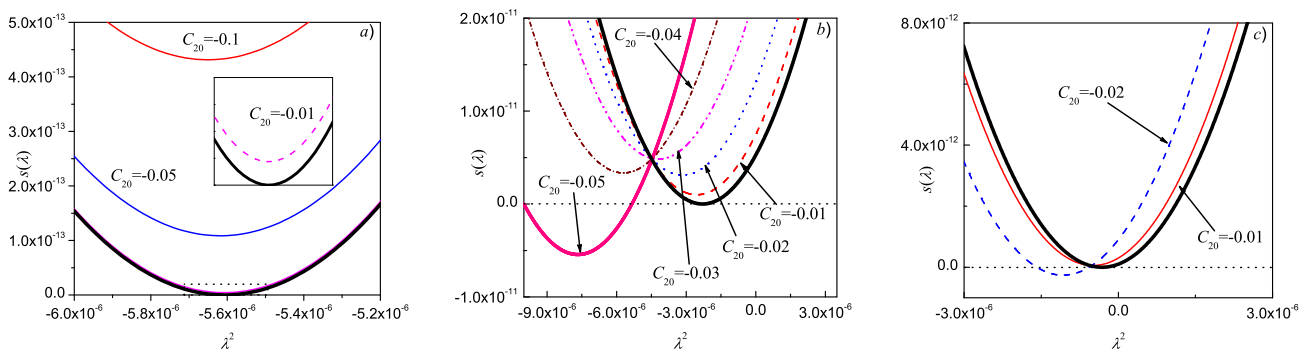

Figure 3: The curves of $s(\lambda)$ for different $S / m, a$ and $C_{20}$. Left: $S / m=0.03 \mathrm{~kg}^{-1} \cdot \mathrm{m}^{2}, a=5$; Middle: $S / m=0.03 \mathrm{~kg}^{-1} \cdot \mathrm{m}^{2}, a=2$; Right: $S / m=0.01 \mathrm{~kg}^{-1} \cdot \mathrm{m}^{2}, a=2$.

$0.03 \mathrm{~kg}^{-1} \cdot \mathrm{m}^{2}$, Fig.3a and $3 \mathrm{~b}$ give the curves of $s(\lambda)$ for different values of the $C_{20}$ term at $a=5$ and $a=2$, respectively. The bold black line corresponds to the curve for $C_{20}=0$ in each plot. In Fig.3a, there are two pairs of complex eigenvalues for $\bar{c}>\bar{b}^{2} / 4$, which is the case for $C_{20} \neq 0$. Therefore, the STO is unstable under the perturbation of the $C_{20}$ term. In Fig.3b, it is found that the STO is still unstable because of $\bar{c}-\bar{b}^{2} / 4>0$ for small value of the $C_{20}$ term. However, when the absolute value of the $C_{20}$ term increases to a critical value (between -0.04 and -0.05), the sign of $\bar{c}-\bar{b}^{2} / 4$ changes from positive to negative, which indicates the STO becomes stable again. Further, if the value of $S / m$ is reduced to 0.01 $\mathrm{kg}^{-1} \cdot \mathrm{m}^{2}$, the critical value of the $C_{20}$ term also decreases (between -0.01 and -0.02), as illustrated in Fig.3c. Numerical simulations are carried out to verify these analysis. For the sake of brevity, details are excluded here.

Concerning the stability of the STO under the joint effects of the $C_{20}$ term and the SRP, following conclusions can be made from Fig. 3 ,

1. When the $C_{20}$ term is small, the STO is unstable under its perturbation. The inclination gradually diffuses away from its initial equilibrium value which 
is close to $90^{\circ}$;

2. For low-altitude motion, the STO can become stable again if the $C_{20}$ term is larger than a critical value. The critical value decreases with the decreasing of the value of $S / m$. This also indicates that for a fixed $C_{20}$ value, the STO tends to be stable for smaller SRP perturbation.

In summary, the introduction of even a very small $C_{20}$ term usually brings instability to the STO. On the other hand, when $C_{20}$ is large enough, the STO becomes stable again.

\section{Heliotropic Orbit}

For the planar Heliotropic orbit, Eq.25 can be satisfied by one of the following conditions

$$
\left\{\begin{array}{l}
H=G, \quad g+h=0 \text { or } \pi \\
H=-G, \quad g-h=0 \text { or } \pi
\end{array}\right.
$$

The first and second cases correspond to a prograde and retrograde orbit, respectively. For both cases, the orbits lie in the asteroid's orbital plane. They are both called heliotropic orbit. The perigee points towards Sun for $g \pm h=0$ and away from the Sun for $g \pm h=\pi$. For the joint effects of zonal harmonic terms and the SRP on the heliotropic orbit, investigations have been made by Lantukh et al. [16]. However, they treat these orbits as EPs of Eq.20. From following discussions, it can be seen that this treatment is inappropriate. In this section, the stability of these orbits is re-investigated and a better criterion is given. 


\subsection{Prograde heliotropic orbit}

For the prograde Heliotropic orbit, we have three conditions: (1) $g+h=0$ or $\pi$, (2) $H=G$, and (3) $\dot{G}=0$. According to Eq 20, when the first two conditions are satisfied, the third condition is automatically satisfied. The condition $g+h=0$ or $\pi$ requires

$$
-\frac{3 C_{20}}{2 L^{3} G^{4}} \pm \frac{3}{2} \tilde{\beta}_{\odot} L \frac{G}{\sqrt{L^{2}-G^{2}}}-n^{\prime}=0
$$

in which the upper and lower signs corresponds to $g+h=0$ and $g+h=\pi$, respectively. The exact value of $G$ can be solved from Eq41. However, Eq41 only indicates $\dot{g}+\dot{h}=0$, but usually $\dot{g}=-\dot{h} \neq 0$. Therefore, heliotropic orbits are not EPs of the system Eq20, and the method of evaluating the stability of the STO in the above section cannot be applied to the heliotropic orbit.

\subsubsection{Orbital eccentricity}

For $C_{20}=0, \mathrm{Eq} 41$ only allows $g+h=0$. This case only allows $G=L \cos \Lambda$, and the eccentricity is obtained as

$$
e=\sin \Lambda
$$

which is a constant for a fixed value of the SRP. However, when $C_{20} \neq 0$, $e$ changes its value for different values of $C_{20}$. Taking $S / m=0.01 \mathrm{~kg}^{-1} \cdot \mathrm{m}^{2}$ and $a_{e}=500 \mathrm{~m}$ as an example, Fig 4 gives the curves of the following functions 


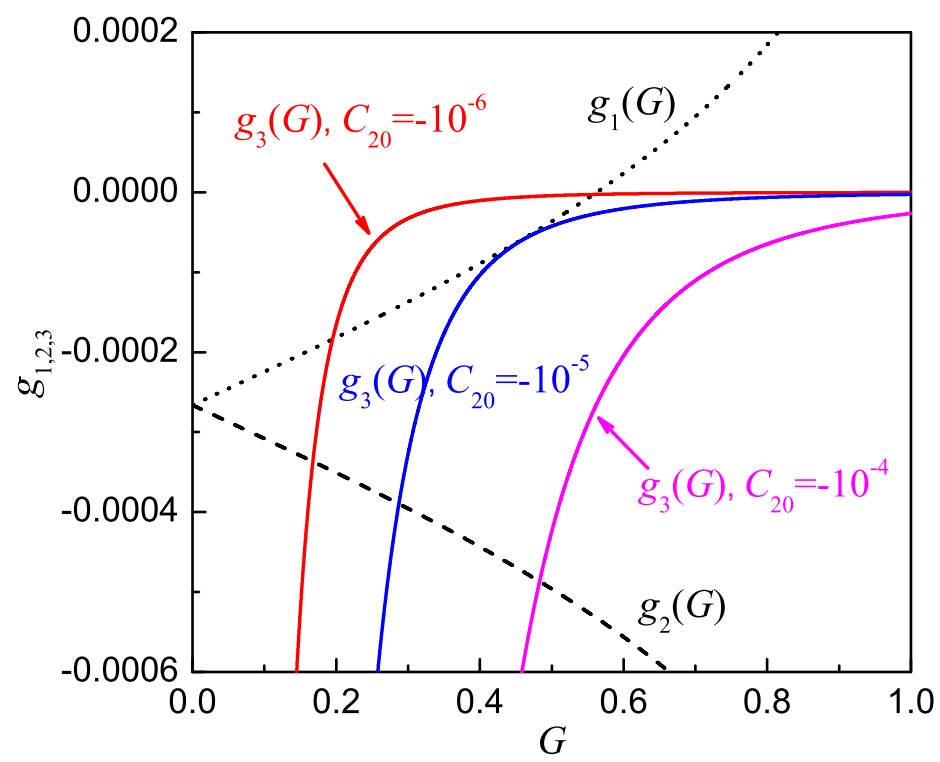

Figure 4: Curves of functions $g_{1}(G), g_{2}(G), g_{3}(G)$ with $S / m=0.01 \mathrm{~kg}^{-1} \cdot \mathrm{m}^{2}$ and $a_{e}=500 \mathrm{~m}$ at different values of the $C_{20}$ term. The unit of the horizontal axis is $\sqrt{L}$

$g_{1}(G), g_{2}(G), g_{3}(G)$ for different values of the $C_{20}$ term

$$
\begin{aligned}
& g_{1}(G)=\frac{3}{2} \tilde{\beta}_{\odot} L \frac{G}{\sqrt{L^{2}-G^{2}}}-n^{\prime}, \\
& g_{2}(G)=-\frac{3}{2} \tilde{\beta}_{\odot} L \frac{G}{\sqrt{L^{2}-G^{2}}}-n^{\prime}, \\
& g_{3}(G)=\frac{3 C_{20}}{2 L^{3} G^{4}} .
\end{aligned}
$$

The condition $g+h=0$ requires $g_{1}=g_{3}$, and the condition $g+h=\pi$ requires $g_{2}=g_{3}$. Obviously, only for very small values of $C_{20}$, the condition $g+h=0$ can be satisfied. Such small $C_{20}$ values are usually impossible for natural celestial bodies. Therefore in practice, the introduction of the $C_{20}$ term only allows the case $g+h=\pi$ in which the perigee points away from the Sun. As a result in the following part, we only consider the case of $g+h=\pi$. 

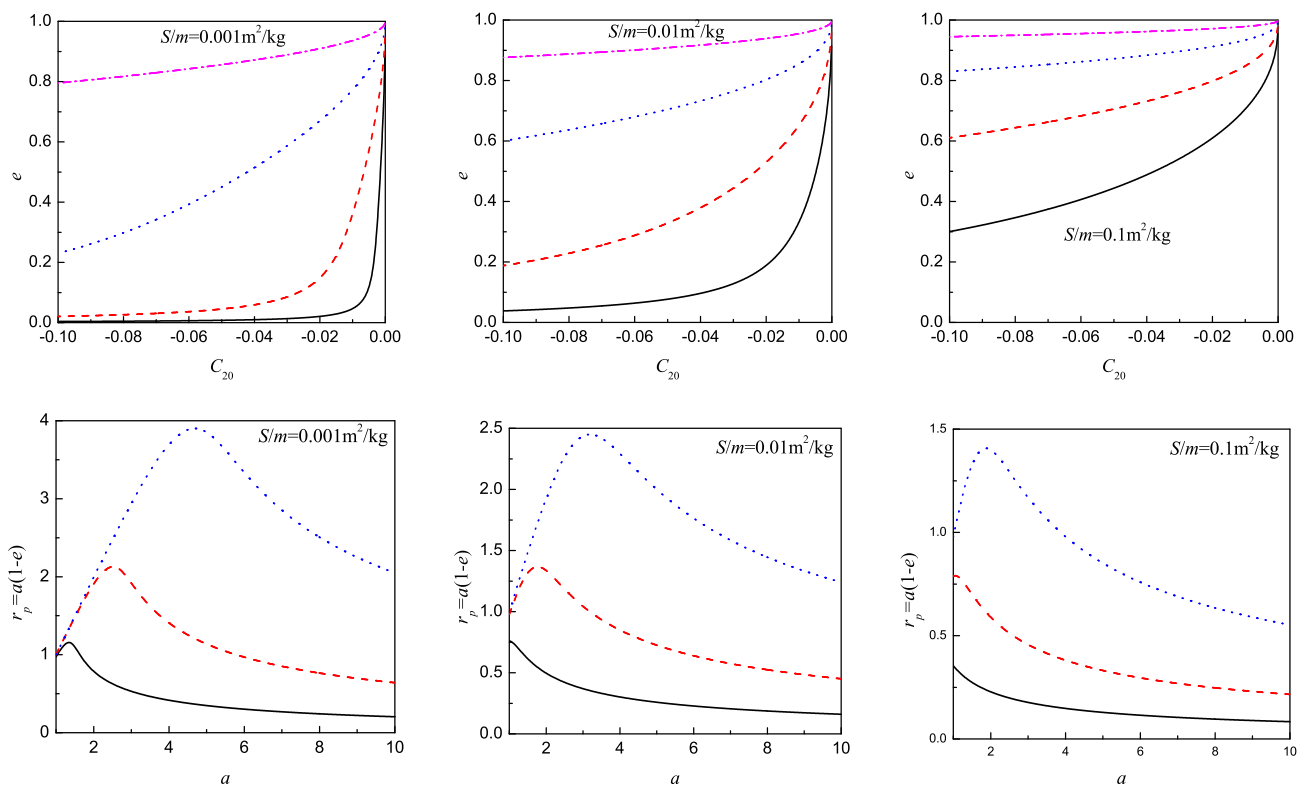

Figure 5: Upper row: the eccentricity of the prograde Heliotropic orbit as a function of the $C_{20}$ term at $S / m=0.001,0.01,0.1 \mathrm{~kg}^{-1} \cdot \mathrm{m}^{2}$, respectively. The black, red dashed, blue dotted and pinkish red dot dashed lines denote $a=2,3,5,10$, respectively. Lower row: variation of the periapsis radius $r_{p}$ as a function of $a$ at different values of $S / m$ and the $C_{20}$ term. The black, red dashed, blue dotted and pinkish red dot dashed lines denote $C_{20}=-0.001,-0.01,-0.1$, respectively.

The upper row of Fig 5 gives the eccentricity of the prograde Heliotropic orbit as a function of the $C_{20}$ term, at $S / m=0.001,0.01,0.1 \mathrm{~kg}^{-1} \cdot \mathrm{m}^{2}$, respectively. From these three frames, it is observed that the orbit eccentricity increases with decreasing $C_{20}$ and increasing $S / m$. The lower row of Fig 5 illustrates the variation of the periapsis radius $r_{p}$ as a function of $a$ at different values of $S / m$ and the $C_{20}$ term. It can be concluded that: (1) For small $C_{20}$ values, the height of the perigee decreases with increasing $a$. (2) For large $C_{20}$ values, the height of the perigee first increases and then decreases. 


\subsubsection{Orbital stability}

As mentioned previously, the prograde Heliotropic orbit is not the EP of Eq.20, as in general $\dot{g} \neq 0, \dot{h} \neq 0$. It can be obtained from the constraint $h+g=\pi$ that

$$
h=\pi-g, \dot{h}=-\dot{g}
$$

which is substituted into the first two equations of Eq.20. Together with the condition of $H=G$, the following relation is acquired

$$
\dot{g}=-\dot{h}=n^{\prime}-\frac{3 C_{20}}{2 L^{3} G^{4}}-\frac{3}{2} \tilde{\beta}_{\odot} \frac{L \sqrt{L^{2}-G^{2}}}{G} \sin ^{2} g=M-N \sin ^{2} g
$$

in which

$$
M=n^{\prime}-\frac{3 C_{20}}{2 L^{3} G^{4}}>0, N=\frac{3}{2} \tilde{\beta}_{\odot} \frac{L \sqrt{L^{2}-G^{2}}}{G}>0
$$

It is discussed in two different cases:

Case 1: If $M>N$, the following integral is obtained

$$
\tan g=\sqrt{\frac{M}{M-N}} \tan \left[\sqrt{M(M-N)}\left(t-t_{0}\right)\right]
$$

where $t_{0}$ is a constant. Obviously, it is a periodic function with a period of

$$
T_{p}=\frac{2 \pi}{\sqrt{M(M-N)}}
$$

Theoretically, the initial value of $g$ can be arbitrarily chosen. Taking $S / m=$ $0.03 \mathrm{~kg}^{-1} \cdot \mathrm{m}^{2}, C_{20}=-0.05$ and $a=4$ as an example, Fig.8a gives the integrals within one period at different initial conditions. Since the curves have the same 
shapes but only different initial phase angles, they actually corresponds to only one solution which is given by Eq47, Fig.8b shows the time history curve of $g+h$ for a nearby orbit around this heliotropic orbit with initial conditions as

$$
g_{0}=\pi / 2, h_{0}=\pi-g_{0}, G_{0}=G^{*}, H_{0}=G^{*} \times 0.999
$$

in which $G^{*}$ is obtained from Eq 41. The value of $g+h$ is librating around $180^{\circ}$, which means that the Heliotropic orbit is stable.

Case 2: If $M<N$, the following integral is obtained from $\mathrm{Eq} 45$

$$
\tan g=\sqrt{\frac{M}{N-M}} \tanh \left[\sqrt{\frac{N-M}{M}}\left(t-t_{0}\right)\right]
$$

where $t_{0}$ is the integral constant and its upper limit is given as

$$
\lim _{t=0}^{+\infty} \tan g=\sqrt{\frac{M}{N-M}}
$$

For different values of $g$, Eq.50 describes completely different trajectories. Taking $S / m=0.03 \mathrm{~kg}^{-1} \cdot \mathrm{m}^{2}, C_{20}=-0.05$ and $a=5$ as an example, Fig.8c gives the evolutions of two trajectories with very close initial values of $g$.

$$
g_{0}=g_{c} \pm \Delta g, g_{c}=\pi-\sin ^{-1} \sqrt{\frac{M}{N}}, \Delta g=0.01 \pi
$$

Fig.8c means that even for infinitely small initial values of $\Delta g$, the final $g$ can approach very different values, which clearly indicates instability. Fig.8d shows an orbit nearby this Heliotropic orbit with initial conditions also given by Eq.49. The value of $g+h$ no longer librates around a value, indicating the instability of the Heliotropic orbit. 

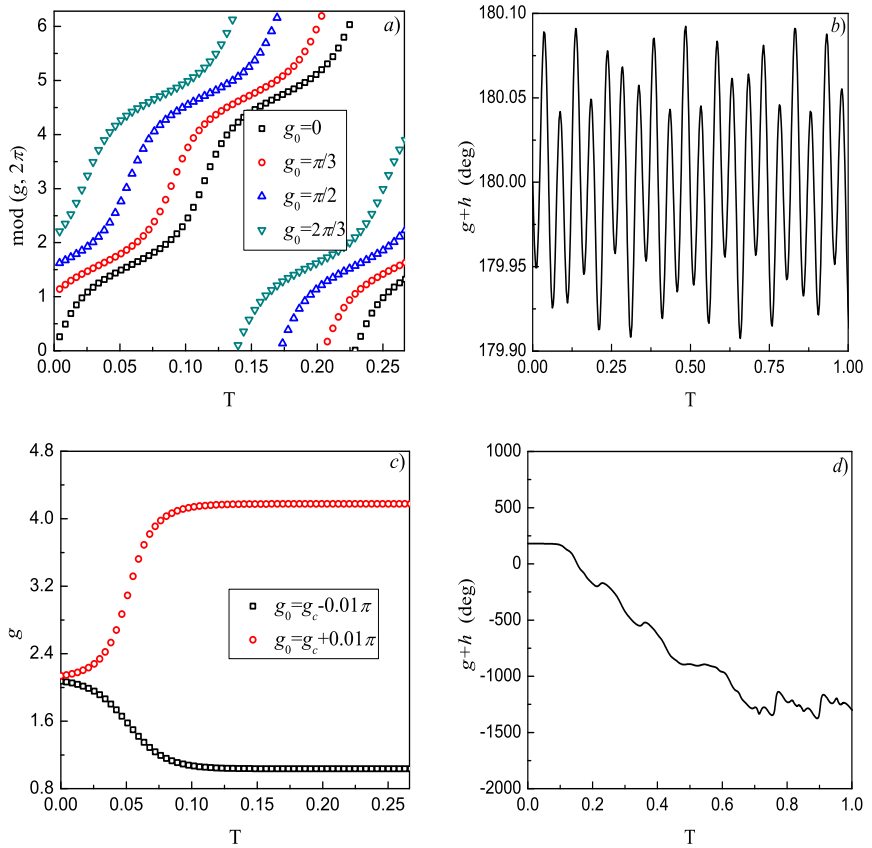

Figure 6: For $a$ ) and $c$ ): Solution of $\mathrm{Eq} 30$ for different initial conditions of $g$; For $b$ ) and $d$ ): The evolution of $g+h$ of an orbit near the Heliotropic orbit. For $a)-d$ ), $S / m=0.03 \mathrm{~kg}^{-1} \cdot \mathrm{m}^{2}$, $C_{20}=-0.05$. For $a$ ) and $\left.b\right), a=4$; for $c$ ) and $\left.d\right), a=5$. 
Table 2: Stability of Heliotropic orbit. Definitions of $M, N, M$ are given by Eq 46 and 57 .

\begin{tabular}{|c|c|c|}
\hline \multirow{4}{*}{ Prograde } & $M / N>1$ (i.e. $a<a_{c}$ ) & stable \\
\cline { 2 - 3 } & $M / N<1$ (i.e. $a>a_{c}$ ) & unstable \\
\hline \multirow{4}{*}{ Retrograde } & $M^{\prime} / N>1$ (i.e. $a<a_{c}^{1}$ ) & stable \\
\cline { 2 - 3 } & $0<M^{\prime} / N<1$ (i.e. $a_{c}^{1}<a<a_{c}^{2}$ ) & unstable \\
\cline { 2 - 3 } & $M^{\prime} / N<0$ (i.e. $a>a_{c}^{2}$ ) & stable \\
\hline
\end{tabular}

To summarize, the value of $M / N$ can be used as a criterion for determining the stability of the heliotropic orbit, which is stable for $M / N>1$ and unstable for $M / N<1$. Given fixed values of $S / m$ and the $C_{20}$ term, there exists a critical value $a_{c}$, and $a<a_{c}$ corresponds to $M / N>1$ while $a>a_{c}$ corresponds to $M / N<1$ (referring to Table2).

\subsection{Retrograde heliotropic orbit}

For the retrograde Heliotropic orbit, the following three conditions should be satisfied: (1) $g-h=0$ or $\pi$, (2) $H=-G$, and (3) $\dot{G}=0$. Similar with the prograde case, the third condition is automatically satisfied if the first two conditions are satisfied. The condition $g-h=0$ or $\pi$ requires

$$
-\frac{3 C_{20}}{2 L^{3} G^{4}} \pm \frac{3}{2} \tilde{\beta}_{\odot} \frac{L G}{\sqrt{L^{2}-G^{2}}}+n^{\prime}=0
$$

with the upper and lower signs for $g-h=0$ and $g-h=\pi$, respectively. The value of $G$ can be solved from $\mathrm{Eq} 53$. However, Eq 53 only means $\dot{g}-\dot{h}=0$, but in general $\dot{g}=\dot{h} \neq 0$. Therefore, retrograde heliotropic orbits are also not EPs of the system Eq.20, and the method of evaluating the stability of the STO cannot be 
applied to them.

\subsubsection{Orbital eccentricity}

For $C_{20}=0, \mathrm{Eq} 53$ only allows $g-h=\pi$, from which $G=L \cos (\Lambda)$. As a result, $e=\sin (\Lambda)$ can be obtained. For $C_{20} \neq 0$, taking $S / m=0.01 \mathrm{~kg}^{-1} \cdot \mathrm{m}^{2}$ and $a_{e}=500 \mathrm{~m}$ as an example, divide $\mathrm{Eq} 53$ into the three following functions $g_{4}(G), g_{5}(G), g_{6}(G)$

$$
\begin{aligned}
& g_{4}(G)=\frac{3}{2} \tilde{\beta}_{\odot} L \frac{G}{\sqrt{L^{2}}-G^{2}}+n^{\prime}, g_{5}(G)=-\frac{3}{2} \tilde{\beta}_{\odot} L \frac{G}{\sqrt{L^{2}-G^{2}}}+n^{\prime}, \\
& g_{3}(G)=\frac{3 C_{20}}{2 L^{3} G^{4}}
\end{aligned}
$$

Therefore, $g_{4}=g_{3}$ and $g_{5}=g_{3}$ correspond to the cases of $g-h=0$ and $g-h=\pi$, respectively. The curves of $g_{4}(G), g_{5}(G)$ have same shapes as those of $g_{1}(G), g_{2}(G)$ in Fig 4 , only with a constant difference of $2 n^{\prime}$. Due to the fact of $g_{4}>0, g_{3}<0$, and $g_{3} \neq g_{4}, g-h=0$ can never happen for the retrograde heliotropic orbit. Moreover, as $g_{5}-g_{2}>0$, the value of $G$ for $g_{5}=g_{3}$ is larger than the value for $g_{2}=g_{3}$, for the same values of $S / m$ and the $C_{20}$ term. As a result, the retrograde heliocentric orbit always has a smaller eccentricity than the prograde orbit. Fig.7 gives the eccentricity (the upper row) and perigee (the lower row) of the retrograde Heliotropic orbit as a function of the $C_{20}$ term, at $S / m=0.001,0.01,0.1 \mathrm{~kg}^{-1} \cdot \mathrm{m}^{2}$ and for different values of $a$, respectively. It can be seen that the eccentricity has similar variations with the prograde case. For the middle and right frames with larger $S / m$ values, they have similar shapes as the middle and right frames of Fig 5. However, for the left frames with a smaller 

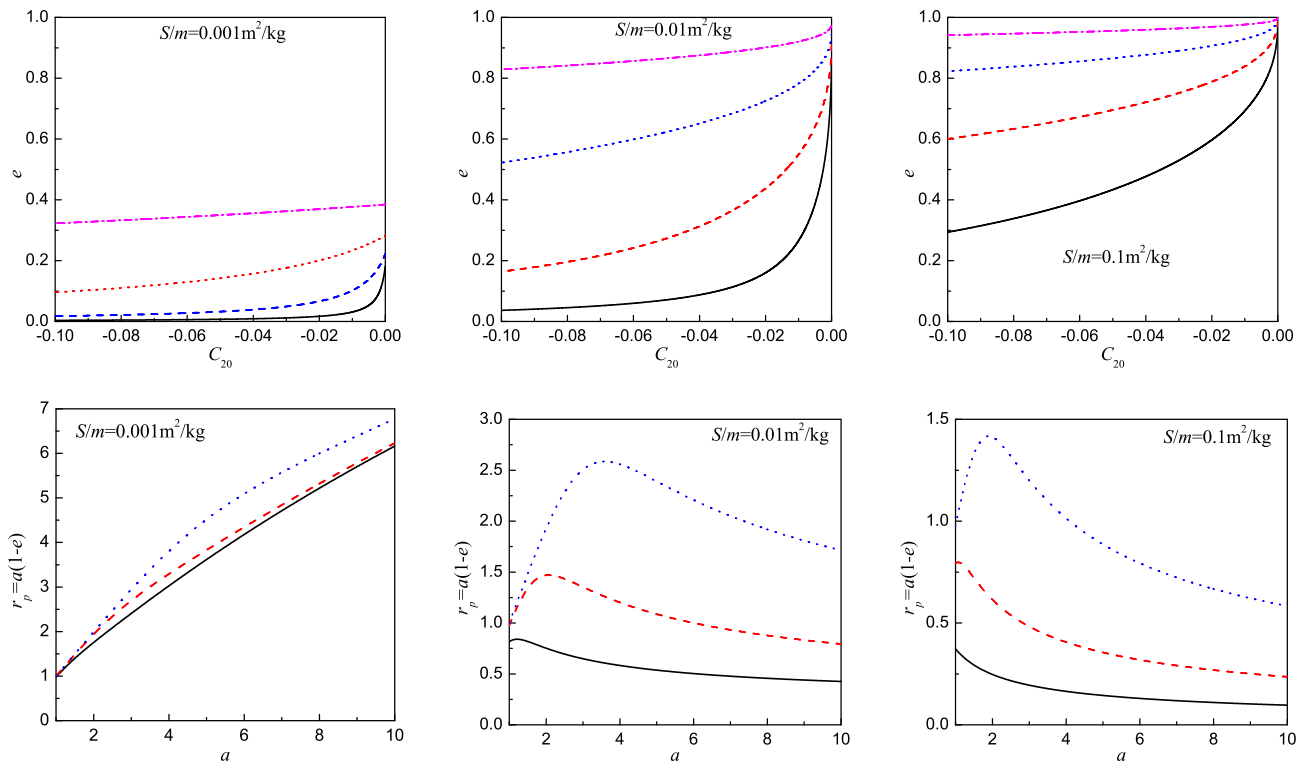

Figure 7: Corresponding to Fig 5 , but for the retrograde heliotropic orbit.

$S / m$ value, the orbit eccentricity for the retrograde case is obviously smaller than the prograde orbit, and the perigee height increases with increasing $a$.

\subsubsection{Orbital stability}

Similarly, the retrograde heliotropic orbit is not the EP of Eq.20. It can be obtained from the constraint $g-h=\pi$ that

$$
h=g-\pi, \dot{h}=\dot{g}
$$

which is substituted into the first two equations of Eq.20. Together with the condition of $H=-G$, the following relation is acquired

$$
\dot{g}=-\frac{3 C_{20}}{2 L^{3} G^{4}}-n^{\prime}-\frac{3}{2} \tilde{\beta}_{\odot} \frac{L \sqrt{L^{2}-G^{2}}}{G} \sin ^{2} g=M^{\prime}-N \sin ^{2} g
$$


in which $N$ has the same definition in $\mathrm{Eq} 45$ and $M^{\prime}$ is defined as

$$
M^{\prime}=-\frac{3 C_{20}}{2 L^{3} G^{4}}-n^{\prime}
$$

whose sign changes with parameters. There are three cases listed as follows: Case 1: If $M^{\prime}<0$, the integral of Eq 56 is obtained

$$
\tan g=\sqrt{\frac{M^{\prime}}{M^{\prime}-N}} \tan \left[-\sqrt{M^{\prime}\left(M^{\prime}-N\right)}\left(t-t_{0}\right)\right]
$$

where $t_{0}$ is an integral constant. Obviously, it is a periodic function with a period of

$$
T_{p}^{\prime}=\frac{2 \pi}{\sqrt{M^{\prime}\left(M^{\prime}-N\right)}}
$$

Case 2: If $M^{\prime}>0$ and $M^{\prime}<N$, the integral is similar to Case 2 of the prograde case and given as

$$
\tan g=\sqrt{\frac{M^{\prime}}{N-M^{\prime}}} \tanh \left[\sqrt{\frac{N-M^{\prime}}{M^{\prime}}}\left(t-t_{0}\right)\right]
$$

where $t_{0}$ is the integral constant and its upper limit is given as

$$
\lim _{t=0}^{+\infty} \tan g=\sqrt{\frac{M^{\prime}}{N-M^{\prime}}}
$$

Case 3: If $M^{\prime}>N>0$, the integral is similar with Case 1 of the prograde case and is given as

$$
\tan g=\sqrt{\frac{M^{\prime}}{M^{\prime}-N}} \tan \left[\sqrt{M^{\prime}\left(M^{\prime}-N\right)}\left(t-t_{0}\right)\right]
$$



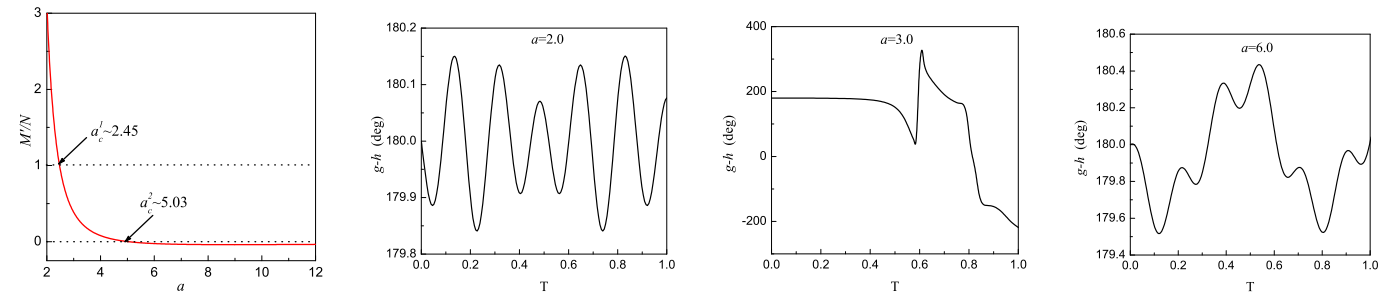

Figure 8: Left: the variation of $M^{\prime} / N$ as the function of $a$ for $S / m=0.01 \mathrm{~kg}^{-1} \cdot \mathrm{m}^{2}$ and $C_{20}=-0.005 ;$ Right (three plots): the evolution of $g-h$ at $a=2,3,6$.

in which $t_{0}$ is also an integral constant. Obviously, it is a periodic function with the same expression as Case 1 for the period.

With $S / m=0.01 \mathrm{~kg}^{-1} \cdot \mathrm{m}^{2}$ and $C_{20}=-0.005$, Fig.8 (upper left) gives the variation of $M^{\prime} / N$ as the function of $a$. From the discussions for cases 1-3, it is known that the orbit is unstable for $0<M^{\prime} / N<1$. Denote the value of $a$ at $M^{\prime} / N=1$ and $M^{\prime} / N=0$ as the critical values $a_{c}^{1}$ and $a_{c}^{2}$, respectively. Taking three values of $a=2,3,6$ locating in the range $\left(0, a_{c}^{1}\right],\left(a_{c}^{1}, a_{c}^{2}\right],\left(a_{c}^{2},+\infty\right]$ respectively, and the following initial conditions

$$
g_{0}=\pi / 3, h_{0}=g_{0}-\pi, G_{0}=G^{*}, H_{0}=-G^{*} \times 0.999
$$

in which $G^{*}$ is obtained from $\mathrm{Eq} 53$, the right three plots of Fig, 8 show the evolutions of $g-h$ of these orbits, whose behavior agree with analysis.

Similar to the prograde case, the value of $M^{\prime} / N$ can be used as a criterion for the stability of the retrograde heliotropic orbit. The orbit is stable for $M^{\prime} / N>1$ and $M^{\prime} / N<0$, and unstable for $0<M^{\prime} / N<1$. Given a certain $S / m$ and the $C_{20}$ term, there exists two critical values $a_{c}^{1}<a_{c}^{2}$ of $a$. The situations of $a<a_{c}^{1}$, 
$a_{c}^{1}<a<a_{c}^{2}$ and $a>a_{c}^{2}$ correspond to $M^{\prime} / N>1,0<M^{\prime} / N<1$ and $M^{\prime} / N<0$, respectively (referring to Table 2).

\section{The global dynamics}

In Sections 3 and 4, two special types of orbits are studied under the joint effects of the $C_{20}$ term and the SRP, including their orbital geometry and stability. In this section, the global dynamics of $\mathrm{Eq} 19$ is discussed qualitatively by applying the Poincaré section, as the system is an autonomous Hamiltonian with two degrees of freedom.

\subsection{Poincaré section}

Firstly, Eq.19 is rewritten as

$$
\begin{aligned}
& \frac{3 C_{20}}{4 L^{3} G^{5}} H^{2}-\left[\frac{3}{4} \frac{\tilde{\beta}_{\odot} L \sqrt{L^{2}-G^{2}}}{G}(\cos (g+h)-\cos (g-h))+n^{\prime}\right] H \\
& -\frac{C_{20}}{4 L^{3} G^{3}}-\frac{1}{2 L^{2}}-\frac{3}{4} \tilde{\beta}_{\odot} L \sqrt{L^{2}-G^{2}}(\cos (g-h)+\cos (g+h)) \\
& -\bar{K}=0
\end{aligned}
$$

in which $\bar{K}$ is the value of the given Hamiltonian. For fixed values of $\bar{K}$ and $G, g, h$, there are two solutions of Eq 64, denoted as $H_{1} \leq H_{2}$. Generally, $H_{1}$ corresponds to a larger orbital inclination and its corresponding orbit can be viewed as retrograde, while $\mathrm{H}_{2}$ corresponds to a prograde orbit. Taking the STO as an example, the Hamiltonian is

$$
\bar{K}=K_{t e r}=-\frac{1}{2 L^{2}}-\frac{C_{20}}{4 L^{3} G^{3}}\left(1-\frac{3 H^{2}}{G^{2}}\right)+\frac{3}{2} \frac{\tilde{\beta}_{\odot} L H \sqrt{L^{2}-G^{2}}}{G}-n^{\prime} H
$$


in which $G, H$ is obtained from Eq,27. According to Eq.25 and Tab.1, the situation of $\sin (g \pm h)=0$ is the focus and the following Poincare sections can be chosen:
A) $h=\pi / 2, H=H_{1} ; \quad$ B) $h=0, H=H_{1}$;
C) $\left.h=-\pi / 2, H=H_{1} ; \quad D\right) h=\pi, H=H_{1}$;
E) $\left.h=\pi / 2, H=H_{2} ; \quad F\right) h=0, H=H_{2}$
G) $\left.h=-\pi / 2, H=H_{2} ; \quad H\right) h=\pi, H=H_{2}$

For all of them, the values of $g, G$ are recorded when the trajectory crosses these sections. Taking section $A$ ) as an example, fixing $h=\pi / 2$, the sample initial values of $g, G$ are selected from $g \in[0,2 \pi)$ and $i \in[0,2 \pi), e \in[0,1)$. For a given value $\bar{K}$ of the Hamiltonian, $H_{1}, H_{2}$ can be determined from Eq 64 and $H=H_{1}$ is taken. The section can be obtained by doing the integration. Since Eq20 has the following symmetry

$$
g, h \Leftrightarrow-g,-h ; g, h \Leftrightarrow g+\pi, h+\pi
$$

only sections $A), B), E), F$ ) need to be studied and the remaining four sections are obtained from this symmetry. Moreover, except some specific orbit, e.g. the STO with $h$ around $\pm \pi / 2$, most orbits have their $h$ ranging between $[0,2 \pi)$. Therefore, only one of the four sections $A)-D$ ) is required for the retrograde orbit. Similarly, only one section is needed from sections $E$ ) $-H$ ) for the prograde orbit. Sections $A$ ) and $E$ ) are used for the following qualitative analysis of the global dynamics. 

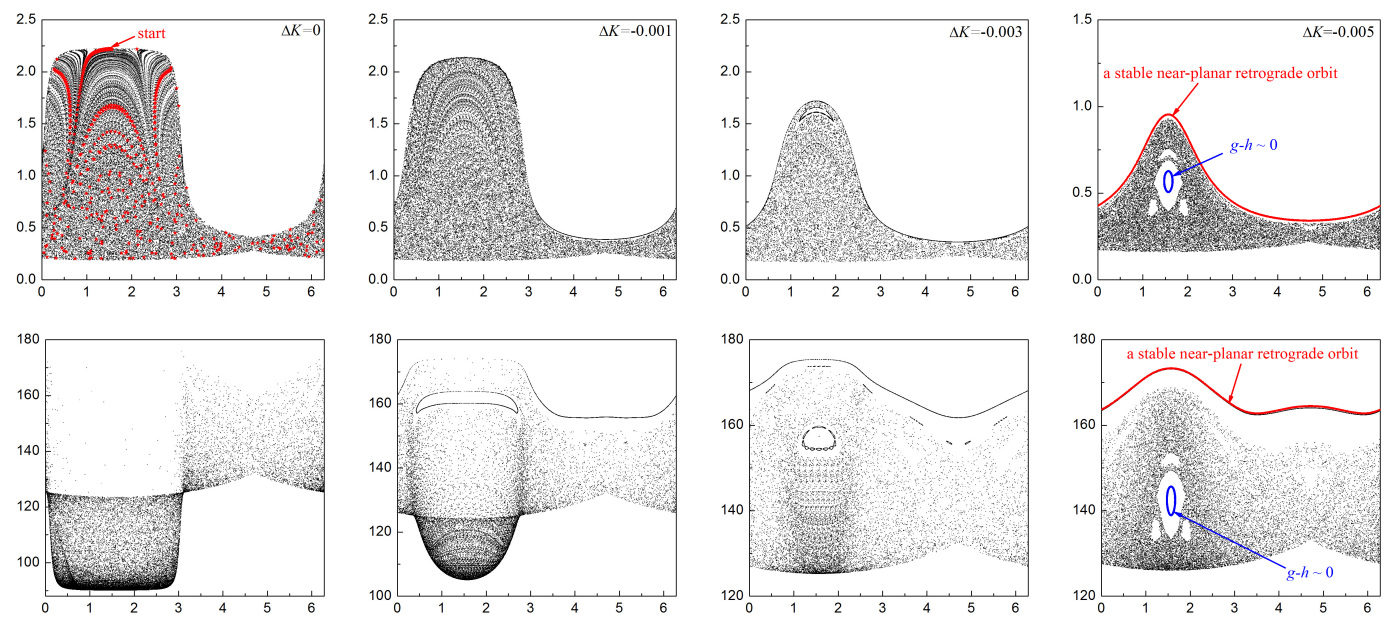

Figure 9: The sections $A$ ) with different Hamiltonians at $C_{20}=-0.01, a=5, S / m=0.03$ $\mathrm{kg}^{-1} \cdot \mathrm{m}^{2}$. The $x$ - and $y$-axis represent $g$ (in $\mathrm{rad}$ ) and $i$ (in $\mathrm{deg}$ ), respectively.

\subsection{The SRP-dominant case}

Taking $C_{20}=-0.01, a=5, S / m=0.03 \mathrm{~kg}^{-1} \cdot \mathrm{m}^{2}$ as an example, the orbital motion is dominated by the SRP, as the $C_{20}$ term is small and the orbital altitude is high.

\subsubsection{Retrograde orbit}

In Fig.9, the upper row gives the sections of $A$ ) with different Hamiltonians $\bar{K}=K_{\text {ter }}+\Delta K$. The lower row illustrates the projection of inclination $i$ on these sections, some parts of which overlap due to the fact that the variable $i$ is not a canonical variable. This phenomenon can be avoided if the canonical variable $H$ is used. However, the variable $H$ depends on both $e$ and $i$. It is not intuitive for observing $i$ and is not applied here.

The upper left plot corresponds to the section of $\bar{K}=K_{t e r}$. On a first look, 
the region with large $G$ values is regular but actually it is chaotic. Starting at the point denoted as 'start' in the figure, the following intersection points on this section gradually disperse to the whole range of the accessible values for $(g, G)$ and the chaotic behavior appears. The divergence rate of $e$ is proportional to the value of the $C_{20}$ term. With the decrease of the Hamiltonian (i.e. the increase of inclination), two regular regions appear on the section. For $\Delta K=-0.005$, they are labelled as red and blue respectively on the section. The red one corresponds to a stable near-planar retrograde orbit, and the blue one has its $g-h$ oscillating around $0^{\circ}$. From the corresponding plot in the lower row, the inclination of the blue orbit ranges between $130^{\circ}$ and $160^{\circ}$. Unfortunately, the orbits in these regular regions all have large $e$ (for $a=5$ ) and collide with the asteroid, meaning that they are of no practical applications.

\subsubsection{Prograde orbit}

Similar to Fig, 9, Fig, 10 gives the sections of $E$ ) with different Hamiltonians $\bar{K}=K_{t e r}+\Delta K$ for the prograde orbit. Similar to the retrograde case, the orbit is generally chaotic for larger values of $\bar{K}$, i.e., larger orbit inclinations. When $\bar{K}$ decreases, i.e., the orbit inclination decreases, stable orbits appear. However, for this study case, these orbits also have large values of $e$ and impact on the asteroid.

\subsubsection{Conclusions}

In the SRP-dominant case, following conclusions can be made from above simulations: (1) When $\mathrm{i}$ is near $90^{\circ}$, the orbital motion is chaotic. The orbit eccentricity vary between 0 and 1 , and the orbit inclination can vary between $0^{\circ}$ and $180^{\circ}$. (2) When the orbit inclination is close to $0^{\circ}$ or $180^{\circ}$, stable prograde or 

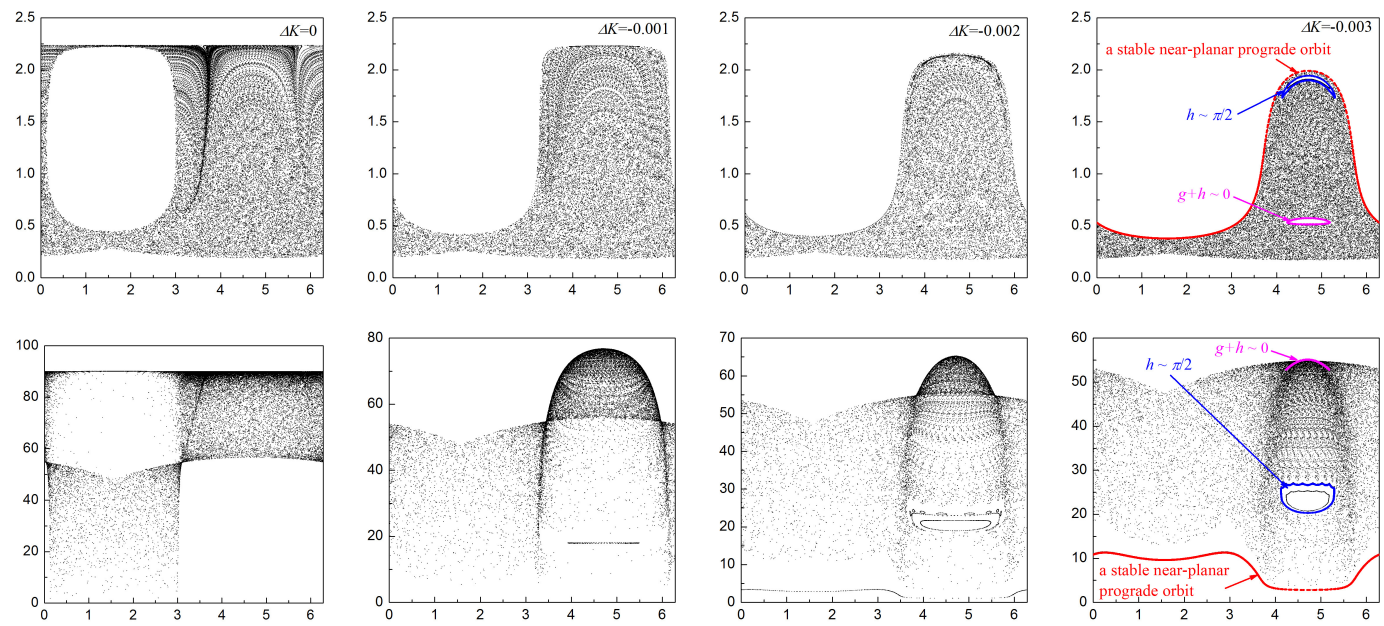

Figure 10: The sections $E$ ) with different Hamiltonians at $C_{20}=-0.01, a=5, S / m=0.03$ $k g^{-1} \cdot m^{2}$. The $x$ - and $y$-axis represent $g$ (in $\operatorname{rad)}$ and $i$ (in $\mathrm{deg}$ ), respectively.

retrograde orbits exist, but they generally have large orbit eccentricities and may collide with the asteroid. As a result, no long-term stable orbits with small orbit eccentricities exist. Nevertheless, the eccentricities of orbits close to the STO with small initial $e$ diverge slowly during the first several orbital periods of the asteroids which usually last for several years, so they can be used for short-duration asteroid missions.

\subsection{The $C_{20}$-dominant case}

Taking $C_{20}=-0.05, a=2, S / m=0.03 \mathrm{~kg}^{-1} \cdot \mathrm{m}^{2}$ as the study case, the orbital motion is dominated by the $C_{20}$ term, as the orbital altitude is very low and close to the asteroid. 

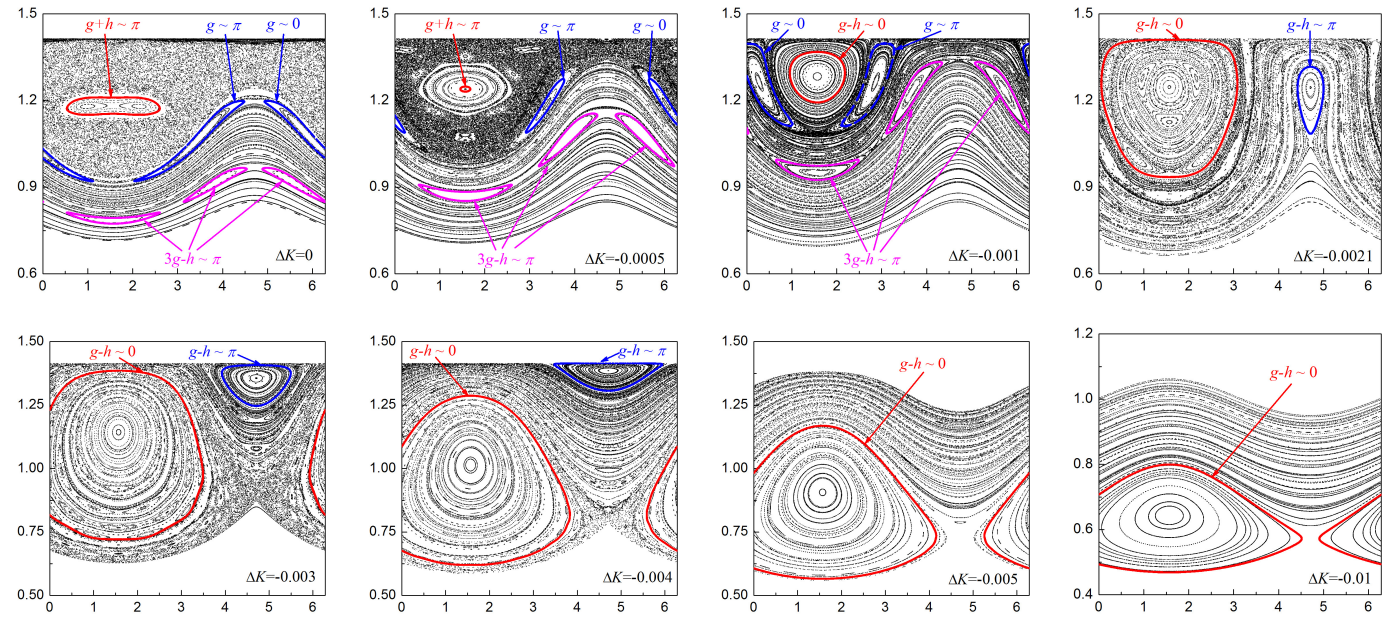

Figure 11: The sections $A$ ) with different Hamiltonians at $C_{20}=-0.01, a=5, S / m=0.03$ $\mathrm{kg}^{-1} \cdot \mathrm{m}^{2}$. The $x$-axis represents $g$ (in $\mathrm{rad}$ ) and $y$-axis represents $G$, respectively.

\subsubsection{Retrograde orbit}

Fig 11 gives the sections of $A$ ) with different Hamiltonians $\bar{K}=K_{\text {ter }}+\Delta K$. The projections of corresponding inclination on these sections are given in Fig.12.

Compared with Fig.9, the chaotic region in Fig.11 and Fig.12 shrinks. The inclination can be divided into two regions at the critical inclination $117^{\circ}$ :

1. For $i \in\left[90^{\circ}, 117^{\circ}\right)$, the orbits are generally chaotic , but two main stable regions exsit around the resonances $\dot{h}=0$ ( $h$ is around $90^{\circ}$ ) and $\dot{g}+\dot{h}=0$ ( $g+h$ is around $180^{\circ}$ ), respectively. However, the resonance $\dot{h}=0$ is not clearly visible in Fig, 11 and Fig,12, so they are magnified and shown in the first two plots of Fig,13. The STO and its nearby orbits ( i.e. the orbits with both $h$ and $g$ librating around $90^{\circ}$ ) only occupy a small region of this stable region. They are denoted as 'Terminator' in Fig. 13. The lower two plots of 

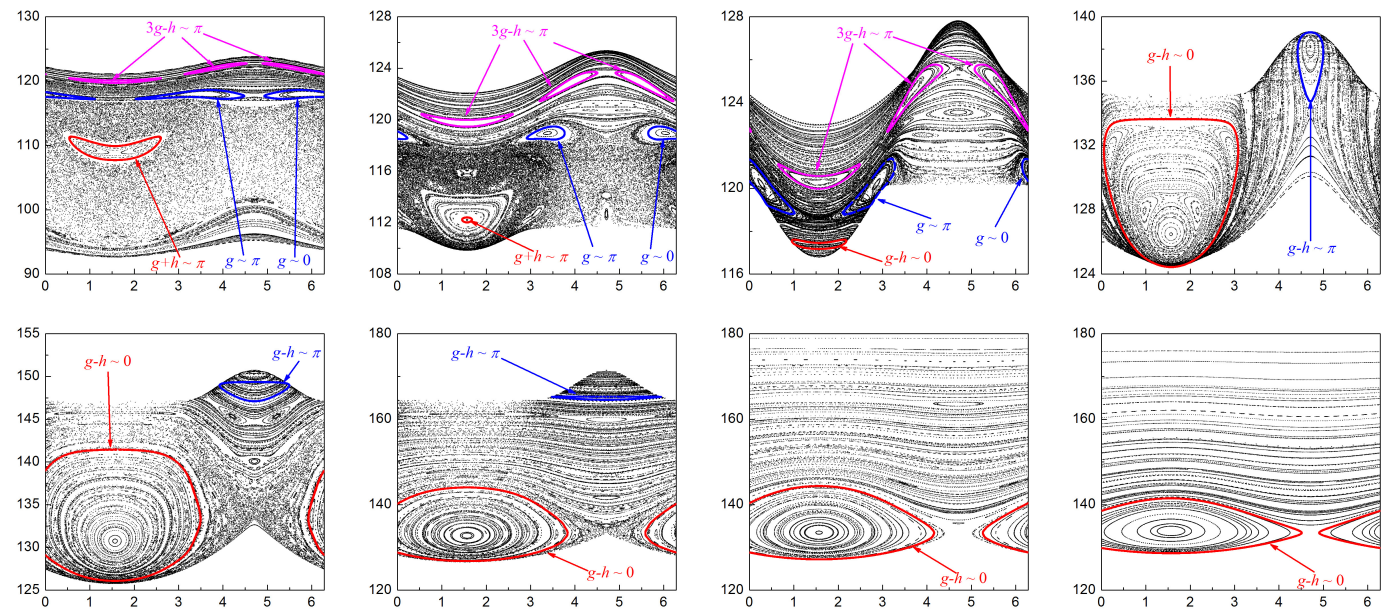

Figure 12: Corresponding to Fig 11, but the y-axis represents the orbit inclination in deg.

Fig. $\sqrt{13}$ also show the evolutions of $\Omega, e, i$ for an example orbit (denoted as 'Example' in the upper two plots of Fig.13) other than the STO type orbit in this region.

2. For $i \in\left(117^{\circ}, 180^{\circ}\right)$, the motion is generally stable. Specifically, two subregions can be distinguished. The first is the resonance region $\dot{g}-\dot{h}=0$, for which $g-h$ is around $0^{\circ}$ and $180^{\circ}$ and the inclination ranges from $125^{\circ}$ to $180^{\circ}$. The orbits in this region either vibrate around $g-h=0$ or $180^{\circ}$, or follow the non-resonant motion. The second is the region near the critical inclination (i.e. $\left.i \in\left(117^{\circ}, 125^{\circ}\right)\right)$, which also includes other resonances, e.g. $\dot{g}=0,3 \dot{g}-\dot{h}=0$. Some parts of this region are chaotic due to the overlap of these resonances.

It is mentioned here that the resonance changes from $\dot{g}+\dot{h}=0$ for $i<117^{\circ}$ to $\dot{g}+\dot{h}=0$ for $i>117^{\circ}$, due to the fact that the sign of $\dot{g}$ changes at the critical 

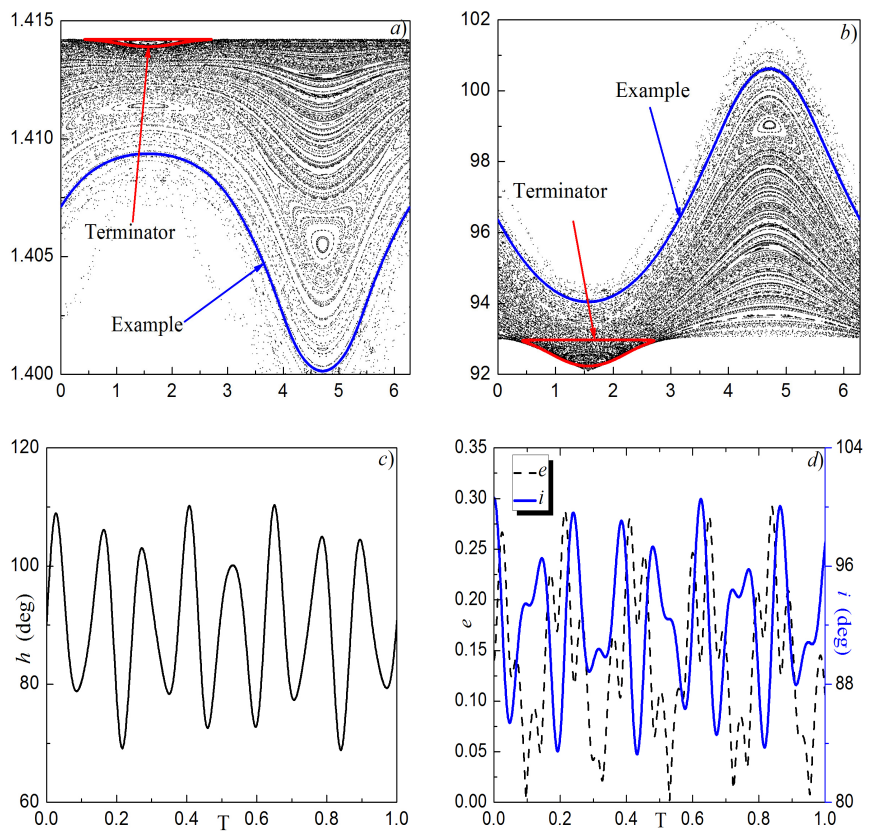

Figure 13: a) The zoom-in of the upper left plot at small $e$ of Fig 11 , b) The zoom-in of the near polar region of Fig 12 , c) the evolution of $\Omega$ of the example orbit; d) the evolution of $e, i$ of the example orbit. The unit of the $x$-axis is the orbital period of the asteroid around Sun. 
inclination.

\subsubsection{Prograde orbit}

Similar to Fig, 11 and Fig, 12 , Fig, 14 and Fig, 15 give the sections of $E$ ) with different Hamiltonians $\bar{K}=K_{t e r}+\Delta K$ and the projections of corresponding inclination $i$ on these sections, respectively.

Similar to the retrograde case, the inclination can also be divided into two regions at the critical inclination $63^{\circ}$ :

1. For $i \in\left(63^{\circ}, 90^{\circ}\right]$, the orbits are generally chaotic, but two main stable regions exist around the resonances $\dot{h}=0$ ( $h$ is around $\left.90^{\circ}\right)$ and $\dot{g}-\dot{h}=0$ ( $g-h$ is around $180^{\circ}$ ), respectively. However, the resonance $\dot{h}=0$ is also not clearly visible in Figs.14 and 15, but the magnified plots are no longer provided. Similarly, the resonance changes from $\dot{g}-\dot{h}=0$ for $i<63^{\circ}$ to $\dot{g}+\dot{h}=0$ for $i>63^{\circ}$, due to the fact that the sign of $\dot{g}$ changes at the critical inclination.

2. For $i \in\left(0,63^{\circ}\right)$, the motion is generally stable. Specifically, two subregions can be distinguished. The first region is dominated by the resonance $\dot{g}+\dot{h}=0$, for which $g+h$ is around $0^{\circ}$ and $180^{\circ}$ and the inclination ranges from $0^{\circ}$ to $55^{\circ}$. The orbits in this region either librate around $g-h \approx 0$ or $180^{\circ}$, or follow the non-resonant motion. The second is the region near the critical inclination (i.e. $\left.i \in\left(55^{\circ}, 63^{\circ}\right]\right)$, which also includes other res-

onances, e.g. $\dot{g}=0,3 \dot{g}+\dot{h}=0,2 \dot{g}+\dot{h}=0$. Some part of this region is chaotic due to the overlap of these resonances. 

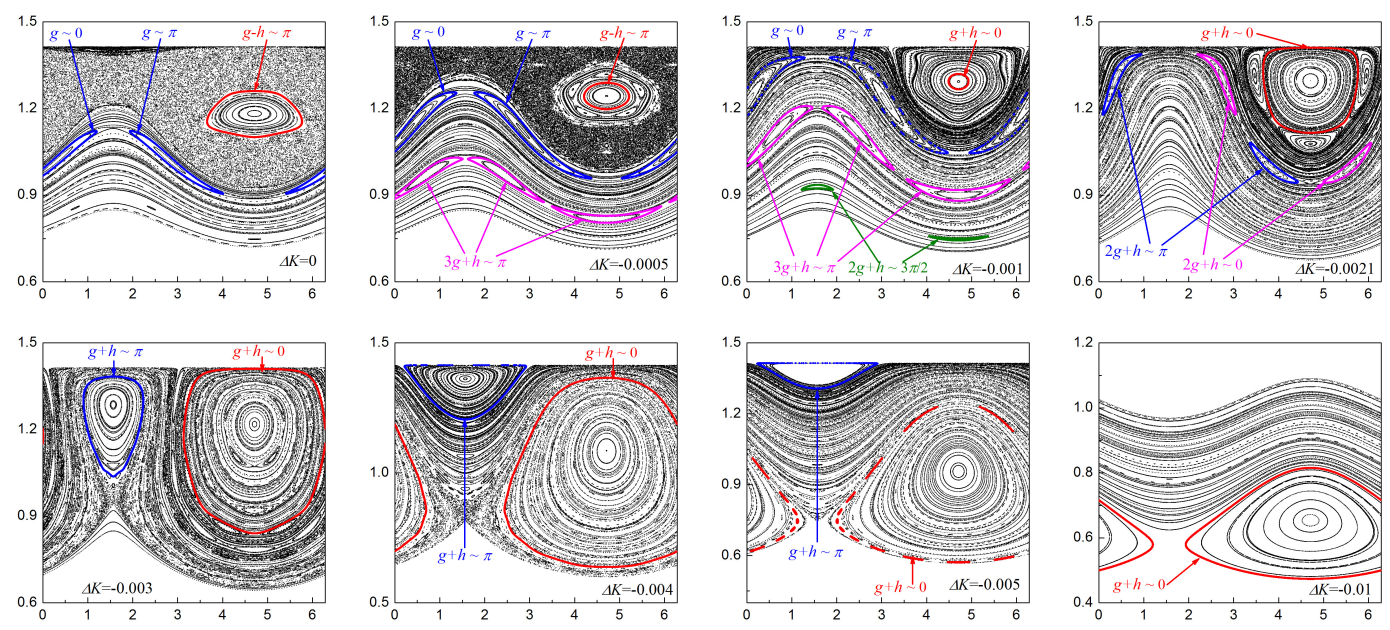

Figure 14: The sections $E$ ) with different Hamiltonians at $C_{20}=-0.01, a=5, S / m=0.03$ $k g^{-1} \cdot m^{2}$. The $x$-axis represents $g$ in rad and $y$-axis represents $G$.
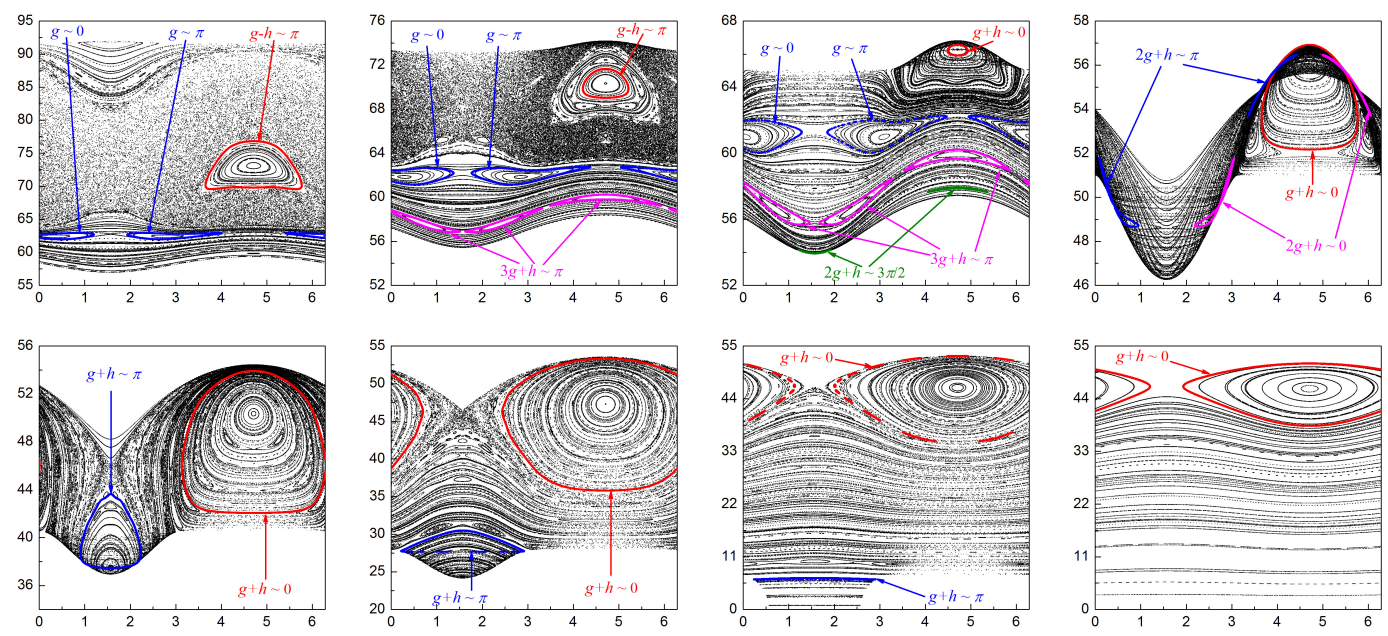

Figure 15: Corresponding to Fig 14, but the $y$-axis represents the orbit inclination in deg. 


\subsection{Conclusions}

For the $C_{20}$-dominant case, following conclusions can be made from above numerical simulations:

1. When $i$ is near $90^{\circ}$, the orbital motion can be stable, but not restricted by the STO type orbits, i.e. both $g$ and $h$ librate around specific values. Nevertheless, the orbital plane of the orbits in the stable region points towards or away from the Sun for $h \approx \pi / 2$ and $h \approx 3 \pi / 2$, respectively, which satisfies $\dot{h}=0$;

2. For $i \in\left(63^{\circ}, 117^{\circ}\right]$, the orbital motion is chaotic, except for the resonance regions $\dot{h}=0, \dot{g}-\dot{h}=0$ and $\dot{g}+\dot{h}=0$;

3. For $i \in\left(0,63^{\circ}\right)$ and $i \in\left(117^{\circ}, 180^{\circ}\right)$, the orbital motion is generally stable.

Depending on the strength of the SRP, there are different extents of variations in the orbit eccentricity. Generally, the variations in the orbit eccentricity for polar orbits are smaller than those for the near-planar orbits. So we can expect practically applicable orbits for polar orbits. Nevertheless, if the strength of the SRP is much smaller than that of the $C_{20}$ term, the extent of variations in the orbit eccentricity caused by the SRP is also small. In this case, we can also expect practical stable near-planar orbits.

\section{Discussion}

The previous analysis is based on the assumption that the asteroid has circular orbit around the Sun. However, most near Earth asteroids usually have large heliocentric eccentricities, between 0.2 and 0.35. Simply ignoring it probably 
affects the accurate analysis of the dynamics. Introducing the asteroid's orbit eccentricity, for the planar motion neglecting the deducing details, the new averaged Hamiltonian is obtained as

$$
\begin{aligned}
K & =-\frac{1}{2 L^{2}}-\frac{C_{20}}{4 L^{3} G^{3}}-\frac{3 \tilde{\beta}_{\odot} a}{8\left(1-e^{2}\right)} e\left\{2 \alpha(1-\cos i) \cos \left(\omega-\Omega+f^{\prime}\right)\right. \\
& +2 \alpha(1+\cos i) \cos \left(\omega+\Omega-f^{\prime}\right) \\
& +2(1-\cos i) e^{\prime}\left[\cos (\omega-\Omega)+\cos \left(\omega-\Omega+2 f^{\prime}\right)\right] \\
& +2(1+\cos i) e^{\prime}\left[\cos \left(\omega+\Omega-2 f^{\prime}\right)+\cos (\omega+\Omega)\right] \\
& +(1-\cos i) e^{\prime 2} / 2\left[\cos \left(\omega-\Omega-f^{\prime}\right)+\cos \left(\omega-\Omega+3 f^{\prime}\right)\right] \\
& \left.+(1+\cos i) e^{\prime 2} / 2\left[\cos \left(\omega+\Omega-3 f^{\prime}\right)+\cos \left(\omega+\Omega+f^{\prime}\right)\right]\right\}
\end{aligned}
$$

in which $e^{\prime}$ and $f^{\prime}$ are the eccentricity and true anomaly of the asteroid's orbit around the Sun, respectively, and $\alpha=1+e^{2} / 2$. If the $C_{20}$ term is ignored, Eq 68 is integrable according to the work by Scheeres [27, 28], Rosengren and Scheeres [24]. However, considering the $C_{20}$ term, the prograde orbit has the following Hamiltonian

$$
\begin{aligned}
K & =-\frac{1}{2 L^{2}}-\frac{C_{20}}{4 L^{3} G^{3}}-\frac{3 \tilde{\beta}_{\odot} a e}{8\left(1-e^{2}\right)}\left\{4 \alpha \cos \left(\tilde{\omega}-f^{\prime}\right)\right. \\
& \left.+4 e^{\prime}\left[\cos \left(\tilde{\omega}-2 f^{\prime}\right)+\cos \tilde{\omega}\right]+e^{\prime 2}\left[\cos \left(\tilde{\omega}-3 f^{\prime}\right)+\cos \left(\tilde{\omega}+f^{\prime}\right)\right]\right\}
\end{aligned}
$$

in which $\tilde{\omega}=\omega+\Omega$. Introducing the following canonical transform

$$
\tilde{g}=\omega+\Omega, \tilde{G}=G-L
$$


the EOM is written as

$$
\begin{aligned}
\dot{\tilde{g}} & =\frac{3 C_{20}}{4 L^{3} \tilde{G}^{4}}-\frac{3 \tilde{\beta}_{\odot} a}{8\left(1-e^{\prime 2}\right)} \frac{\partial e}{\partial \tilde{G}}\left\{4 \alpha \cos \left(\tilde{g}-f^{\prime}\right)\right. \\
& \left.+4 e^{\prime}\left[\cos \left(\tilde{g}-2 f^{\prime}\right)+\cos \tilde{g}\right]+e^{\prime 2}\left[\cos \left(\tilde{g}-3 f^{\prime}\right)+\cos \left(\tilde{g}+f^{\prime}\right)\right]\right\} \\
\dot{\tilde{G}} & =-\frac{3 \beta_{\odot} L \sqrt{L^{2}-\tilde{G}^{2}}}{8\left(1-e^{\prime 2}\right)}\left\{4 \alpha \sin \left(\tilde{g}-f^{\prime}\right)\right. \\
& \left.+4 e^{\prime}\left[\sin \left(\tilde{g}-2 f^{\prime}\right)+\sin \tilde{g}\right]+e^{\prime 2}\left[\sin \left(\tilde{g}-3 f^{\prime}\right)+\sin \left(\tilde{g}+f^{\prime}\right)\right]\right\}
\end{aligned}
$$

For any initial condition, Eq.71 can be integrated and the Poincaré section is generated by recording the canonical pair $\tilde{g}, \tilde{G}$ when $f^{\prime}=2 k \pi, k \in \mathrm{Z}$ is satisfied. Fig.18 gives some examples with $S / m=0.01 \mathrm{~kg}^{-1} \cdot \mathrm{m}^{2}, a_{e}=1 \mathrm{~km}, a=3 \mathrm{~km}$ at $e^{\prime}=0,0.1,0.5,0.7$, respectively. From left to right, the $C_{20}$ term takes values of $-0.001,-0.005,-0.01,-0.05$, respectively. The following conclusions can be made from these figures:

1. Comparison between different rows of figures indicates that the phase space is significantly changed when $e^{\prime}$ is large, in form of the resonance splitting (the resonance $\tilde{g}-M^{\prime}=\pi$ into sub-resonances, arising from the high-order terms [17]), and the expansion of the chaotic region. In the chaotic region, some analytical solutions for the planar motion such as the one developed by Casanova et al. [4] is no longer valid;It also can be seen that $e^{\prime}$ needs to be considered if it has large values. The incorporation of $e^{\prime}$ in the modelling and analysis is carried out by our recent research [8].

2. For a certain SRP, Comparison between different rows of figures indicates that the regular region expands with the increase of the $C_{20}$ term, when 
the planar motion is dominated by the $C_{20}$ term with the SRP as a small perturbation.

For the retrograde orbit, its Hamiltonian is written as

$$
\begin{aligned}
K & =-\frac{1}{2 L^{2}}-\frac{C_{20}}{4 L^{3} G^{3}}-\frac{3 \tilde{\beta}_{\odot} a e}{8\left(1-e^{\prime 2}\right)}\left\{4 \alpha \cos \left(\tilde{\omega}^{\prime}+f^{\prime}\right)\right. \\
& \left.+4 e^{\prime}\left[\cos \left(\tilde{\omega}^{\prime}+2 f^{\prime}\right)+\cos \tilde{\omega}\right]+e^{\prime 2}\left[\cos \left(\tilde{\omega}^{\prime}+3 f^{\prime}\right)+\cos \left(\tilde{\omega}^{\prime}-f^{\prime}\right)\right]\right\}
\end{aligned}
$$

in which $\tilde{\omega}^{\prime}=\omega-\Omega$. Similar conclusions as Fig. 16 can be made, as it is symmetric w.r.t. Eq 69 in $f^{\prime}$.

\section{Conclusions}

This study investigates the long-term dynamical behavior of orbital motion around asteroid, under the joint effects of the $C_{20}$ term and the SRP. Two types of special orbits are studied, namely the STO and the Heliotropic orbit. For the former one, the following observations are made:

1. For a certain SRP, the introduction of the $C_{20}$ term brings instability to the STO and makes its inclination larger than $90^{\circ}$, referring to Fig. 2 for details;

2. For a certain SRP, although the $C_{20}$ term makes the STO unstable, the orbit becomes stable again if the $C_{20}$ term is large enough, referring to Fig 3 for details.

For the Heliotropic orbit, our study shows that: 

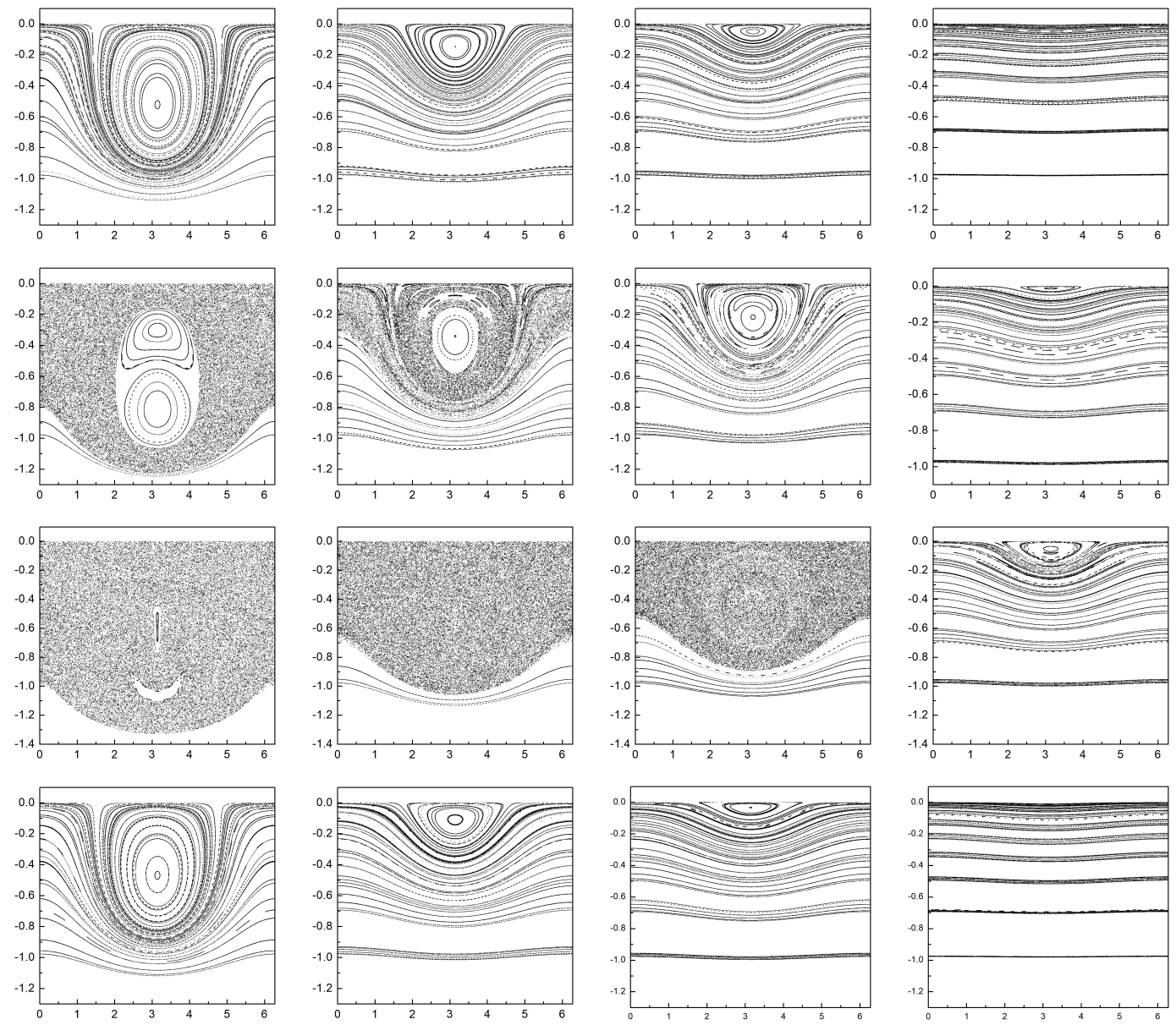

Figure 16: The Poincaré section of Eq71 for $e^{\prime}=0,0.1,0.5,0.7$ and $C_{20}=$ $-0.001,-0.005,-0.01,-0.05$. 
1. For the prograde orbit, the introduction of the $C_{20}$ term changes its $g+h$ from 0 to $\pi$ (referring to Fig, 4) and varies its eccentricity (referring to Fig.5 and 7);

2. For the prograde orbit, there is a critical value $a_{c}$, and the orbit is stable for $a<a_{c}$ and unstable for $a>a_{c}$ (referring to Fig 6); for the retrograde orbit, there exist two critical values $a_{c}^{1}<a_{c}^{2}$, and the orbit is stable for $a<a_{c}^{1}$ or $a>a_{c}^{2}$ and unstable for $a_{c}^{1}<a<a_{c}^{2}$.

By applying the Poincaré section, the global dynamics is investigated for two situations: the SRP dominates over the perturbative $C_{20}$ term and the $C_{20}$ term dominates the perturbative SRP. Following conclusions are made:

1. For the SRP-dominating case, even small value of the $C_{20}$ term can bring global chaos to the phase space. Nevertheless, when $i$ is close to $0^{\circ}$ or $180^{\circ}$, stable orbits with large orbit eccentricity exist (referring to Figs 9 and 10). They collide with the asteroid due to the large orbit eccentricity. In contrast, the near polar orbits are unstable but with a slow divergence rate of the eccentricity. These orbits have potential applications for asteroid missions before the orbit eccentricity grows too large. In summary, smallsized particles or probes cannot stay around the asteroid for long time with the dominant SRP effect;

2. For the $C_{20}$-dominating case, the motion is generally stable (referring to Figs 12 and 15 for $i \in\left(0,63^{\circ}\right)$ and $i \in\left(117^{\circ}, 180^{\circ}\right)$. For $i \in\left(63^{\circ}, 90^{\circ}\right)$ and $\left(90^{\circ}, 117^{\circ}\right)$, the stable regions exist around resonances $\dot{g}-\dot{h}=0$ and $\dot{g}+\dot{h}=0$, respectively. Near polar region includes stable resonance region 
$\dot{h}=0$ (namely pointing towards or away from the Sun), among which STO exists (referring to Fig.13). These near-polar stable orbits are practically applicable due to their small eccentricity.

Finally, the effect of the asteroid's orbital eccentricity $e^{\prime}$ around the Sun on the long-term orbital motion around the asteroid is discussed for the planar motion. Comparing with the system without considering the asteroid's orbit eccentricity, the large value $e^{\prime}$ breaks the integrability and also changes the phase space structure.

The method developed here can also be applied to study the orbital motion around large celestial bodies, for which situation the solar gravitation needs to be considered. Our research simplifies the dynamics in terms of ignoring highorder zonal harmonics, the obliquity of the asteroid and the tesseral harmonics, which have influence on the dynamics quantitatively or qualitatively. Although the tesseral harmonics only have short-term effect but will affect the long-term property of motion close to the resonance region. In our previous work [15], the combined effects of the $C_{20}$ and $C_{22}$ terms on the long term orbital motion around the asteroid was investigated. After taking the SRP into account, new characteristics of the dynamics need to be evaluated for asteroids with different rotation rates.

\section{Acknowledgements}

This work is funded by the Natural Science Foundation of China with No. 11773017 and No. 11673072. 


\section{References}

[1] Broschart S.B., Scheeres D.J., Villac B.F., 2014, Advances in the Astronautical Sciences, 135, 1685

[2] Broschart S.B., Lantoine G., Grebow D.J., 2014, Celestial Mechanics and Dynamical Astronomy, 120, 195

[3] Burns J.A., Lamy P.L., Soter S., 1979, Icarus, 40, 1

[4] Casanova D., Petit A., Lematre A., 2015, Celestial Mechanics and Dynamical Astronomy, 123, 223.

[5] Chanut T.G.G., Albaae S., Prado A.F.B.A., Carruba V., 2015, Monthly Notices of the Royal Astronomical Society, 470, 2687

[6] Colombo C., Lücking C., McInnes C.R., 2010, Acta Astronautica, 81, 137

[7] García Y.D., Sanchez C.J.P., McInnes C.R., 2015, Journal of Guidance, Control and Dynamics, 38, 280

[8] Feng J.L., Hou X.Y., 2018, Advances in Sapce Research, 62, 2649

[9] Giancotti M., Campagnola S., 2014, Celestial Mechanics and Dynamical Astronomy, 120, 269

[10] Hamilton D.P., Burns J.A., 1991, Icarus, 92, 118

[11] Hamilton D.P., Burns J.A., 1992, Icarus, 96, 43

[12] Hedman M.M., Burt J.A., Burns J.A., Tiscareno M.S., Icarus, 210, 284 
[13] Hesar S.G., Scheeres D.J., McMahon J.W., 2017, Journal of Guidance, Control, and Dynamics, 40, 81

[14] Hou X.Y., Liu L., 2013, Celestial Mechanics and Dynamical Astronomy, 116,241

[15] Hou X.Y., Xin X.S., Feng J.L., 2018, Communications in Nonlinear Science and Numerical Simulation, 40, 81

[16] Lantukh D., Rusell R.P., Broschart S., 2015, Celestial Mechanics and Dynamical Astronomy, 121, 171

[17] Lemaítre, A., 1984, Celestial Mechanics, 32, 109

[18] Liu Y.Y., Villac B., 2010, Advances in the Astronautical Sciences, 136, 285

[19] McDonnell J.A.M., Alexander W.M., Burton W.M., et al., 1986, Nature, 321, 338

[20] Mignard F., 1982, Icarus, 49, 347

[21] Mignard F., Hénon M., 1984, Celestial Mechanics, 33, 239

[22] Peale S.J., 1966, Journal of Geophysical Research, 71, 911

[23] Richter K., Keller H.U., 1995, on the stability of dust particle orbits around cometary nuclei, Icarus, 114, 355

[24] Rosengren A.J., Scheeres D.J., 2014, Celestial Mechanics and Dynamical Astronomy, 118, 197 
[25] Russell R.P., Lantukh D., Broschart S.B., 2016, Journal of Guidance Control and Dynamics, 39, 1

[26] Sfair R., Winter S.M., Giuliatti, Mourão D.C., Winter O.C., 2009, Monthly Notices of the Royal Astronomical Society, 395, 2157

[27] Scheeres D.J., 1999, Journal of the Astronautical Science, 47, 25

[28] Scheeres D.J., 2007, 20th International Symposium on Space Flight Dynamics, Sep. 24-28

[29] Scheeres D.J., 2012, Springer, New York

[30] Scheeres D.J., Sutter B.M., Rosengren A.J., 2013, Advances in the Astronautical Sciences, 148, 3263

[31] Schildknecht T., Musci R., Flohrer T., 2008, Advances in Space Research, 41,1039

[32] Valk S., Lemaítre A., Anselmo L., 2008, Icarus, 41, 1077

[33] Vokrouhlický D., Bottke W.F., Chesley S.R., Scheeres D.J., Statler T.S., 2015, Asteroids IV, University of the Arizona Press, 509

[34] Xin X.S., Scheeres D.J., Hou, X.Y., 2016, Celestial Mechanics and Dynamical Astronomy, 126, 405

[35] Zhao C.Y., Zhang M.J., Wang H.B., Zhang W., Xiong J.N., 2013, Advances in Space Research, 52, 677 


\section{Appendix}

$$
\begin{aligned}
& a_{11}=-\frac{3}{4} \tilde{\beta}_{\odot} \frac{L G}{\sqrt{L^{2}-G^{2}}}\left[\left(1-\frac{H}{G}\right) \sin (g-h)+\left(1+\frac{H}{G}\right) \sin (g+h)\right] \\
& +\frac{3}{4} \tilde{\beta}_{\odot} \frac{L H \sqrt{L^{2}-G^{2}}}{G^{2}}[\sin (g-h)-\sin (g+h)] \\
& a_{12}=\frac{3}{4} \tilde{\beta}_{\odot} \frac{L G}{\sqrt{L^{2}-G^{2}}}\left[\left(1-\frac{H}{G}\right) \sin (g-h)-\left(1+\frac{H}{G}\right) \sin (g+h)\right] \\
& -\frac{3}{4} \tilde{\beta}_{\odot} \frac{L H \sqrt{L^{2}-G^{2}}}{G^{2}}[\sin (g-h)-\sin (g+h)] \\
& a_{13}=\frac{3 C_{20}}{2 L^{3} G^{5}}\left(\frac{15 H^{2}}{G^{2}}-2\right)+\frac{3}{2} \tilde{\beta}_{\odot} \frac{L^{3} H}{G^{3} \sqrt{L^{2}-G^{2}}}[\cos (g-h)-\cos (g+h)] \\
& +\frac{3}{4} \tilde{\beta}_{\odot} \frac{L^{3}}{\left(L^{2}-G^{2}\right)^{3 / 2}}\left[\left(1-\frac{H}{G}\right) \cos (g-h)+\left(1+\frac{H}{G}\right) \cos (g+h)\right] \\
& a_{14}=-\frac{15 C_{20} H}{2 L^{3} G^{6}}-\frac{3}{4} \tilde{\beta}_{\odot} \frac{L^{3}}{G^{2} \sqrt{L^{2}-G^{2}}}[\cos (g-h)-\cos (g+h)] \\
& a_{21}=-\frac{3}{4} \tilde{\beta}_{\odot} \frac{L \sqrt{L^{2}-G^{2}}}{G}[\sin (g-h)-\sin (g+h)] \\
& a_{22}=\frac{3}{4} \tilde{\beta}_{\odot} \frac{L \sqrt{L^{2}-G^{2}}}{G}[\sin (g-h)+\sin (g+h)] \\
& a_{23}=-\frac{15 C_{20} H}{2 L^{3} G^{6}}-\frac{3}{4} \tilde{\beta}_{\odot} \frac{L^{3}}{G^{2} \sqrt{L^{2}-G^{2}}}[\cos (g-h)-\cos (g+h)] \\
& a_{24}=\frac{3 C_{20}}{2 L^{3} G^{5}} \\
& a_{31}=-\frac{3}{4} \tilde{\beta}_{\odot} L \sqrt{L^{2}-G^{2}}\left[\left(1-\frac{H}{G}\right) \cos (g-h)+\left(1+\frac{H}{G}\right) \cos (g+h)\right] \\
& a_{32}=\frac{3}{4} \tilde{\beta}_{\odot} L \sqrt{L^{2}-G^{2}}\left[\left(1-\frac{H}{G}\right) \cos (g-h)-\left(1+\frac{H}{G}\right) \cos (g+h)\right] \\
& a_{33}=\frac{3}{4} \tilde{\beta}_{\odot} \frac{L G}{\sqrt{L^{2}-G^{2}}}[\sin (g-h)+\sin (g+h)] \\
& -\frac{3}{4} \tilde{\beta}_{\odot} \frac{L^{3} H}{\tilde{G}^{2} \sqrt{L^{2}-G^{2}}}[\sin (g-h)-\sin (g+h)] \\
& a_{34}=\frac{3}{4} \tilde{\beta}_{\odot} \frac{L \sqrt{L^{2}-G^{2}}}{G}[\sin (g-h)-\sin (g+h)]
\end{aligned}
$$




$$
\begin{aligned}
a_{41}= & \frac{3}{4} \tilde{\beta}_{\odot} L \sqrt{L^{2}-G^{2}}\left[\left(1-\frac{H}{G}\right) \cos (g-h)-\left(1+\frac{H}{G}\right) \cos (g+h)\right] \\
a_{42}= & -\frac{3}{4} \tilde{\beta}_{\odot} L \sqrt{L^{2}-G^{2}}\left[\left(1-\frac{H}{G}\right) \cos (g-h)+\left(1+\frac{H}{G}\right) \cos (g+h)\right] \\
a_{43}= & -\frac{3}{4} \tilde{\beta}_{\odot} \frac{L G}{\sqrt{L^{2}-G^{2}}}[\sin (g-h)-\sin (g+h)] \\
& +\frac{3}{4} \tilde{\beta}_{\odot} \frac{L^{3} H}{G^{2} \sqrt{L^{2}-G^{2}}}[\sin (g-h)+\sin (g+h)] \\
a_{44}= & -\frac{3}{4} \tilde{\beta}_{\odot} \frac{L \sqrt{L^{2}-G^{2}}}{G}[\sin (g-h)+\sin (g+h)]
\end{aligned}
$$

